\title{
Order in Implication Zroupoids
}

\author{
Juan M. CORNEJO and Hanamantagouda P. SANKAPPANAVAR
}

\begin{abstract}
The variety I of implication zroupoids (using a binary operation $\rightarrow$ and a constant 0 ) was defined and investigated by Sankappanavar in [7], as a generalization of De Morgan algebras. Also, in [7, several new subvarieties of $\mathbf{I}$ were introduced, including the subvariety $\mathbf{I}_{\mathbf{2}, \mathbf{0}}$, defined by the identity: $x^{\prime \prime} \approx x$, which plays a crucial role in this paper. Some more new subvarieties of $\mathbf{I}$ are studied in [3] that includes the subvariety SL of semilattices with a least element 0; and an explicit description of semisimple subvarieties of $\mathbf{I}$ is given in [5].

It is a well known fact that there is a partial order (denote it by $\sqsubseteq$ ) induced by the operation $\wedge$, both in the variety $\mathbf{S L}$ of semilattices with a least element and in the variety DM of De Morgan algebras. As both SL and DM are subvarieties of $\mathbf{I}$ and the definition of partial order can be expressed in terms of the implication and the constant, it is but natural to ask whether the relation $\sqsubseteq$ on $\mathbf{I}$ is actually a partial order in some (larger) subvariety of I that includes both SL and DM.

The purpose of the present paper is two-fold: Firstly, a complete answer is given to the above mentioned problem. Indeed, our first main theorem shows that the variety $\mathbf{I}_{\mathbf{2}, \mathbf{0}}$ is a maximal subvariety of $\mathbf{I}$ with respect to the property that the relation $\sqsubseteq$ is a partial order on its members.. In view of this result, one is then naturally led to consider the problem of determining the number of non-isomorphic algebras in $\mathbf{I}_{\mathbf{2}, \mathbf{0}}$ that can be defined on an $n$-element chain (herein called $\mathbf{I}_{\mathbf{2}, \mathbf{0}}$-chains), $n$ being a natural number. Secondly, we answer this problem in our second main theorem which says that, for each $n \in \mathbb{N}$, there are exactly $n$ nonisomorphic $\mathbf{I}_{\mathbf{2}, \mathbf{0}}$-chains of size $n$.
\end{abstract}

\section{Introduction}

The widely known fact that Boolean algebras can be defined using only implication and a 'constant was extended to De Morgan algebras in [7]. The crucial role played by a certain identity, called (I), led Sankappanavar, in 2012, to define and investigate, the variety I of implication zroupoids (I-zroupoids) generalizing De Morgan algebras. Also, in [7, he introduced several new 'subvarieties of $\mathbf{I}$ and found some relationships among those subvarieties. One of the subvarieties of $\mathbf{I}$, called $\mathbf{I}_{\mathbf{2}, \mathbf{0}}$, defined by the identity: $x^{\prime \prime} \approx x$ and studied in [7, plays a crucial role in 'this paper. In [3], we introduce several more new subvarieties of $\mathbf{I}$, including the subvariety $\mathbf{S L}$ 'which is term-equivalent to the (well known) variety of $\vee$-semilattices with a least element 0 , and describe further relationships among the subvarities of $\mathbf{I}$. An explicit description of semisimple subvarieties of $\mathbf{I}$ is given in [5].

It is also a well known fact that there is a partial order induced by the operation $\wedge$, both in the variety $\mathbf{S L}$ of semilattices with a least element and in the variety DM of De Morgan algebras. As both $\mathbf{S L}$ and $\mathbf{D M}$ are subvarieties of $\mathbf{I}$ and the defintion of partial order can be expressed in terms of the implication and constant, it is but natural to ask whether the relation $\sqsubseteq$ (now defined) on $\mathbf{I}$ is actually a partial order in some (larger) subvariety of $\mathbf{I}$ that includes both SL and DM. 
The purpose of the present paper is two-fold: Firstly, a complete answer is given to the above mentioned problem. Indeed, our first main theorem shows that the variety $\mathbf{I}_{\mathbf{2}, \mathbf{0}}$ is a maximal subvariety of $\mathbf{I}$ with respect to the property that the relation $\sqsubseteq$, defined by:

$$
x \sqsubseteq y \text { if and only if }\left(x \rightarrow y^{\prime}\right)^{\prime}=x \text {, for } x, y \in \mathbf{A} \text { and } \mathbf{A} \in \mathbf{I},
$$

is a partial order. In view of this result, one is then naturally led to consider the problem of determining the number of non-isomorphic algebras in $\mathbf{I}_{\mathbf{2}, \mathbf{0}}\left(\mathbf{I}_{\mathbf{2}, \mathbf{0}^{-}}\right.$-chains) that can be defined on an $n$-element set, $n$ being a nutural number. Secondly, we answer this problem in our second main result which says that, for each $n \in \mathbb{N}$, there are exactly $n$ nonisomorphic $\mathbf{I}_{2,0^{-}}$-chains of size $n$.

\section{Preliminaries}

In this section we recall some definitions and results from [3], 5] and [7] that will be needed for this paper. Basic references are [1] and [2].

Definition 2.1 [] $A$ groupoid with zero (zroupoid, for short) is an algebra $\mathbf{A}=\langle A, \rightarrow, 0\rangle$, where $\rightarrow$ is a binary operation and 0 is a constant. A zroupoid $\mathbf{A}=\langle A, \rightarrow, 0\rangle$ is an implication zroupoid (I-zroupoid, for short) if the following identities hold in $\mathbf{A}$, where $x^{\prime}:=x \rightarrow 0$ :

(I) $(x \rightarrow y) \rightarrow z \approx\left[\left(z^{\prime} \rightarrow x\right) \rightarrow(y \rightarrow z)^{\prime}\right]^{\prime}$

$\left(\mathrm{I}_{0}\right) 0^{\prime \prime} \approx 0$.

The variety of I-zroupoids is denoted by $\mathbf{I}$.

In this paper we use the characterizations of De Morgan algebras, Kleene algebras and Boolean algebras (see [7]), and semilattices with least element 0 (see [3]), as definitions.

Definition 2.2 An implication zroupoid $\mathbf{A}=\langle A, \rightarrow, 0\rangle$ is a De Morgan algebra (DM-algebra, for short) if $\mathbf{A}$ satisfies the axiom:

$(\mathrm{DM})(x \rightarrow y) \rightarrow x \approx x$.

A DM-algebra $\mathbf{A}=\langle A, \rightarrow, 0\rangle$ is a Kleene algebra (KL-algebra, for short) if $\mathbf{A}$ satisfies the axiom:

$\left(\mathrm{KL}_{1}\right)(x \rightarrow x) \rightarrow(y \rightarrow y)^{\prime} \approx x \rightarrow x$

or, equivalently,

$\left(\mathrm{KL}_{2}\right)(y \rightarrow y) \rightarrow(x \rightarrow x) \approx x \rightarrow x$.

A DM-algebra $\mathbf{A}=\langle A, \rightarrow, 0\rangle$ is a Boolean algebra (BA-algebra, for short) if $\mathbf{A}$ satisfies the axiom:

(BA) $x \rightarrow x \approx 0^{\prime}$.

An implication zroupoid $\mathbf{A}=\langle A, \rightarrow, 0\rangle$ is a semilattice with 0 (SL-algebra, for short) if $\mathbf{A}$ satisfies the axioms:

$(\mathrm{SM} 1) x^{\prime} \approx x$

(SM2) $x \rightarrow y \approx y \rightarrow x$. (Commutativity). 
We denote by $\mathbf{D M}, \mathbf{K L}, \mathbf{B A}$ and $\mathbf{S L}$, respectively, the variety of $\mathbf{D M}$-algebras, KL-algebras, BA-algebras, and SL-algebras.

We recall from [7] the definition of another subvariety of $\mathbf{I}$, namely $\mathbf{I}_{2,0}$, which plays a fundamental role in this paper.

Definition $2.3 \mathbf{I}_{2,0}$ denotes the subvariety of $\mathbf{I}$ defined by the identity:

$$
x^{\prime \prime} \approx x .
$$

We note that DM, KL, BA and $\mathbf{S L}$ are all subvarieties of $\mathbf{I}_{2,0}$ (see [7] and [3]).

Lemma 2.4 [7, Theorem 8.15] Let A be an I-zroupoid. Then the following are equivalent:

(a) $0^{\prime} \rightarrow x \approx x$

(b) $x^{\prime \prime} \approx x$

(c) $\left(x \rightarrow x^{\prime}\right)^{\prime} \approx x$

(d) $x^{\prime} \rightarrow x \approx x$

Lemma 2.5 [7] Let $\mathbf{A} \in \mathbf{I}_{2,0}$. Then

(a) $x^{\prime} \rightarrow 0^{\prime} \approx 0 \rightarrow x$

(b) $0 \rightarrow x^{\prime} \approx x \rightarrow 0^{\prime}$.

Several identities true in $\mathbf{I}_{2,0}$ are given in [3], [5] and [7]. Some of those that are needed for this paper are listed in the next lemma, which also presents some new identities of $\mathbf{I}_{2,0}$ that will be useful later in this paper. The proof of the lemma is given in the Appendix.

Lemma 2.6 Let $\mathbf{A} \in \mathbf{I}_{2,0}$. Then $\mathbf{A}$ satisfies:

(1) $\left(x \rightarrow 0^{\prime}\right) \rightarrow y \approx\left(x \rightarrow y^{\prime}\right) \rightarrow y$

(2) $\left(0 \rightarrow x^{\prime}\right) \rightarrow(y \rightarrow x) \approx y \rightarrow x$

(3) $(y \rightarrow x)^{\prime} \approx(0 \rightarrow x) \rightarrow(y \rightarrow x)^{\prime}$

(4) $\left[x \rightarrow(y \rightarrow x)^{\prime}\right]^{\prime} \approx(x \rightarrow y) \rightarrow x$

(5) $(y \rightarrow x) \rightarrow y \approx(0 \rightarrow x) \rightarrow y$

(6) $0 \rightarrow x \approx 0 \rightarrow(0 \rightarrow x)$

(7) $0 \rightarrow\left[(0 \rightarrow x) \rightarrow\left(0 \rightarrow y^{\prime}\right)^{\prime}\right] \approx 0 \rightarrow(x \rightarrow y)$

(8) $\left[x^{\prime} \rightarrow(0 \rightarrow y)\right]^{\prime} \approx(0 \rightarrow x) \rightarrow(0 \rightarrow y)^{\prime}$

(9) $0 \rightarrow(0 \rightarrow x)^{\prime} \approx 0 \rightarrow x^{\prime}$

(10) $0 \rightarrow\left(x^{\prime} \rightarrow y\right)^{\prime} \approx x \rightarrow\left(0 \rightarrow y^{\prime}\right)$

(11) $\left[\left(x \rightarrow 0^{\prime}\right) \rightarrow y\right]^{\prime} \approx(0 \rightarrow x) \rightarrow y^{\prime}$ 
(12) $0 \rightarrow\left[(0 \rightarrow x) \rightarrow y^{\prime}\right] \approx x \rightarrow\left(0 \rightarrow y^{\prime}\right)$

(13) $0 \rightarrow(x \rightarrow y) \approx x \rightarrow(0 \rightarrow y)$

(14) $(x \rightarrow y) \rightarrow y^{\prime} \approx y \rightarrow(x \rightarrow y)^{\prime}$

(15) $\left(x^{\prime} \rightarrow y\right) \rightarrow\left[(0 \rightarrow z) \rightarrow x^{\prime}\right] \approx(0 \rightarrow y) \rightarrow\left[(0 \rightarrow z) \rightarrow x^{\prime}\right]$

(16) $0 \rightarrow\left(x \rightarrow y^{\prime}\right)^{\prime} \approx 0 \rightarrow\left(x^{\prime} \rightarrow y\right)$

(17) $x \rightarrow\left(y \rightarrow x^{\prime}\right) \approx y \rightarrow x^{\prime}$

(18) $[(0 \rightarrow x) \rightarrow y] \rightarrow x \approx y \rightarrow x$

(19) $[0 \rightarrow(x \rightarrow y)] \rightarrow x \approx(0 \rightarrow y) \rightarrow x$

(20) $(0 \rightarrow x) \rightarrow(0 \rightarrow y) \approx x \rightarrow(0 \rightarrow y)$

(21) $x \rightarrow y \approx x \rightarrow(x \rightarrow y)$

(22) $[\{x \rightarrow(0 \rightarrow y)\} \rightarrow z]^{\prime} \approx z \rightarrow[(x \rightarrow y) \rightarrow z]^{\prime}$

(23) $[0 \rightarrow(x \rightarrow y)] \rightarrow y^{\prime} \approx y \rightarrow(x \rightarrow y)^{\prime}$

$(24) x \rightarrow[(y \rightarrow z) \rightarrow x]^{\prime} \approx(0 \rightarrow y) \rightarrow\left[x \rightarrow(z \rightarrow x)^{\prime}\right]$

$(25) 0 \rightarrow[(0 \rightarrow x) \rightarrow y] \approx x \rightarrow(0 \rightarrow y)$

(26) $x \rightarrow(y \rightarrow x)^{\prime} \approx\left(y \rightarrow 0^{\prime}\right) \rightarrow x^{\prime}$

(27) $\left[\left(x^{\prime} \rightarrow y\right) \rightarrow(z \rightarrow x)^{\prime}\right] \rightarrow[(y \rightarrow z) \rightarrow x] \approx(y \rightarrow z) \rightarrow x$

(28) $\left[\left\{0 \rightarrow(x \rightarrow y)^{\prime}\right\} \rightarrow\left(0 \rightarrow y^{\prime}\right)^{\prime}\right]^{\prime} \approx 0 \rightarrow(x \rightarrow y)^{\prime}$

(29) $\left[[0 \rightarrow\{(x \rightarrow y) \rightarrow z\}] \rightarrow\{0 \rightarrow(y \rightarrow z)\}^{\prime}\right]^{\prime} \approx 0 \rightarrow\{(x \rightarrow y) \rightarrow z\}$

(30) $\left[x \rightarrow(0 \rightarrow y)^{\prime}\right]^{\prime} \approx x^{\prime} \rightarrow\left(y \rightarrow 0^{\prime}\right)^{\prime}$

(31) $[(0 \rightarrow x) \rightarrow y]^{\prime} \approx y \rightarrow(x \rightarrow y)^{\prime}$

(32) $\left[x \rightarrow\left(y \rightarrow 0^{\prime}\right)^{\prime}\right]^{\prime} \approx x^{\prime} \rightarrow(0 \rightarrow y)^{\prime}$

(33) $(x \rightarrow y)^{\prime} \rightarrow(0 \rightarrow x)^{\prime} \approx y^{\prime} \rightarrow x^{\prime}$

(34) $(0 \rightarrow x)^{\prime} \rightarrow(0 \rightarrow y)^{\prime} \approx 0 \rightarrow\left(x^{\prime} \rightarrow y^{\prime}\right)$

(35) $\left[(x \rightarrow y)^{\prime} \rightarrow\left\{y \rightarrow(x \rightarrow y)^{\prime}\right\}^{\prime}\right]^{\prime} \approx(x \rightarrow y)^{\prime}$

(36) $\left[\{(0 \rightarrow x) \rightarrow y\} \rightarrow(x \rightarrow y)^{\prime}\right]^{\prime} \approx(0 \rightarrow x) \rightarrow y$

(37) $\left[\left\{x \rightarrow(y \rightarrow x)^{\prime}\right\} \rightarrow x\right]^{\prime} \approx x \rightarrow(y \rightarrow x)^{\prime}$. 


\section{Partial order in Implication Zroupoids}

Let $\mathbf{A}=\langle A ; \rightarrow, 0\rangle \in \mathbf{I}$. We define the operations $\wedge$ and $\vee$ on $\mathbf{A}$ by:

- $x \wedge y:=\left(x \rightarrow y^{\prime}\right)^{\prime}$,

- $x \vee y:=\left(x^{\prime} \wedge y^{\prime}\right)^{\prime}$.

Note that the above definition of $\wedge$ is a simultaneous generalization of the $\wedge$ operation of De Morgan algebras and that of SL (= semilattices with least element 0$)$. It is, of course, well known that the meet operation induces a partial order on both DM and SL, which naturally leads us to the following definition of a binary relation $\sqsubseteq$ on algebras in $\mathbf{I}$.

Definition 3.1 Let $\mathbf{A} \in \mathbf{I}$. We define the relation $\sqsubseteq$ on $A$ as follows:

$$
x \sqsubseteq y \text { if and only if } x \wedge y=x \quad\left(\text { equivalently, }\left(x \rightarrow y^{\prime}\right)^{\prime}=x\right) .
$$

For $a, b \in A$, we write

- $a \sqsubset b$ if $a \sqsubseteq b$ and $a \neq b$,

- $a \sqsupseteq b$ if $b \sqsubseteq a$, and

- $a \sqsupset b$ if $a \sqsupseteq b$ and $a \neq b$.

We already know from [3] that $\langle A ; \wedge, \vee\rangle$ is a lattice if and only if $\mathbf{A}$ is a De Morgan Algebra, implying that $\sqsubseteq$ is a partial order on $A$. We know (see [3]) that $\sqsubseteq$ is also a partial order on algebras in SL. This fact led us naturally to consider the possibility of the existence of a subvariety $\mathbf{V}$ of $\mathbf{I}$, containing both $\mathbf{S L}$ and $\mathbf{D M}$, such that, for every algebra $\mathbf{A}$ in $\mathbf{V}$, the relation $\sqsubseteq$ on $\mathbf{A}$ is actually a partial order.

In this section we will prove our first main result which says that the subvariety $\mathbf{I}_{\mathbf{2}, \mathbf{0}}$, is a maximal subvariety of $\mathbf{I}$ with respect to the property that the relation $\sqsubseteq$ is a partial order on every member of that variety. To achieve this end, we need to, first, prove that $\sqsubseteq$ is indeed a partial order on every member of $\mathbf{I}_{\mathbf{2}, \mathbf{0}}$, which will be done using the following lemmas.

Lemma 3.2 Let $\mathbf{A} \in \mathbf{I}_{2,0}$. Then the relation $\sqsubseteq$ is antisymmetric on $\mathbf{A}$.

Proof Let $a, b \in A$ such that $a \sqsubseteq b$ and $b \sqsubseteq a$. Let $c \in A$ be arbitrary. Then, using (I) and the hypothesis, one observes that $(c \rightarrow a) \rightarrow b^{\prime}=\left[(b \rightarrow c) \rightarrow\left(a \rightarrow b^{\prime}\right)^{\prime}\right]^{\prime}=[(b \rightarrow c) \rightarrow a]^{\prime}$. Consequently,

$$
(c \rightarrow a) \rightarrow b^{\prime}=[(b \rightarrow c) \rightarrow a]^{\prime}, \text { where } c \in A .
$$

Hence,

$$
\begin{aligned}
a^{\prime} & =(a \wedge b)^{\prime} & & \text { by hypothesis } \\
& =\left(a \rightarrow b^{\prime}\right)^{\prime \prime} & & \text { by definition of } \wedge \\
& =a \rightarrow b^{\prime} & & \\
& =\left(a^{\prime} \rightarrow a\right) \rightarrow b^{\prime} & & \text { using Lemma 2.4(1(d) } \\
& =\left[\left(b \rightarrow a^{\prime}\right) \rightarrow a\right]^{\prime} & & \text { from (3.1) with } c=a^{\prime} \\
& =\left[\left(b \rightarrow a^{\prime}\right)^{\prime \prime} \rightarrow a\right]^{\prime} & & \\
& =\left(b^{\prime} \rightarrow a\right)^{\prime} & & \text { by hypothesis, }
\end{aligned}
$$


and, therefore,

(3.2) $a^{\prime}=\left(b^{\prime} \rightarrow a\right)^{\prime}$.

Now,

$$
\begin{aligned}
b^{\prime} & =\left[b \rightarrow a^{\prime}\right]^{\prime \prime} & & \text { by hypothesis } \\
& =b \rightarrow a^{\prime} & & \\
& =\left(0 \rightarrow a^{\prime \prime}\right) \rightarrow\left(b \rightarrow a^{\prime}\right) & & \text { by Lemma } 2.6 \text { (2) with } x=a^{\prime}, y=b \\
& =(0 \rightarrow a) \rightarrow\left(b \rightarrow a^{\prime}\right)^{\prime \prime} & & \\
& =(0 \rightarrow a) \rightarrow b^{\prime} & & \text { by hypothesis. }
\end{aligned}
$$

Thus,

$(3.3) b^{\prime}=(0 \rightarrow a) \rightarrow b^{\prime}$.

Therefore,

$$
\begin{array}{rlrl}
a^{\prime} & =\left[b^{\prime} \rightarrow a\right]^{\prime} & & \text { from (3.2) } \\
& =[(b \rightarrow 0) \rightarrow a]^{\prime} & \\
& =(0 \rightarrow a) \rightarrow b^{\prime} & & \text { from (3.1) } \\
& =b^{\prime} & \text { bith } c=0
\end{array}
$$

Consequently, we have that $a=a^{\prime \prime}=b^{\prime \prime}=b$, thus proving that $\sqsubseteq$ is antisymmetric on $\mathbf{A}$.

Now, we turn to proving the transitivity of the relation $\sqsubseteq$. For this, we need the following lemmas. The proof of the following (technical) lemma is given in the Appendix.

Lemma 3.3 Let $\mathbf{A} \in \mathbf{I}_{2,0}$ with $a, b \in A$ such that $a \sqsubseteq b$. Let $d \in A$ be arbitrary. Then

(1) $\left(0 \rightarrow a^{\prime}\right) \rightarrow b=a^{\prime} \rightarrow b$

(2) $b \rightarrow a^{\prime}=(0 \rightarrow b) \rightarrow a^{\prime}$

(3) $b \rightarrow a^{\prime}=a^{\prime}$

(4) $0 \rightarrow\left(a^{\prime} \rightarrow b\right)=0 \rightarrow a$

(5) $[(b \rightarrow d) \rightarrow a]^{\prime}=(d \rightarrow a) \rightarrow b^{\prime}$

(6) $(0 \rightarrow d) \rightarrow a^{\prime}=\left[\left\{d \rightarrow\left(0 \rightarrow b^{\prime}\right)\right\} \rightarrow a\right]^{\prime}$

(7) $a \rightarrow\left[\left(a^{\prime} \rightarrow d\right) \rightarrow\left\{(0 \rightarrow a) \rightarrow b^{\prime}\right\}\right]=(0 \rightarrow d) \rightarrow a^{\prime}$

(8) $a \rightarrow\left[(d \rightarrow a) \rightarrow b^{\prime}\right]=a \rightarrow(d \rightarrow a)^{\prime}$

(9) $[0 \rightarrow(b \rightarrow d)] \rightarrow a=(0 \rightarrow d) \rightarrow a$

(10) $[b \rightarrow(a \rightarrow d)] \rightarrow a=(0 \rightarrow d) \rightarrow a$

(11) $b \rightarrow\left(0 \rightarrow a^{\prime}\right)=0 \rightarrow a^{\prime}$

(12) $\left[(d \rightarrow a) \rightarrow b^{\prime}\right]^{\prime}=(b \rightarrow d) \rightarrow a$

(13) $a^{\prime} \rightarrow b=b^{\prime} \rightarrow a$

(14) $\left(d \rightarrow a^{\prime}\right) \rightarrow b=\left(d \rightarrow 0^{\prime}\right) \rightarrow\left(a^{\prime} \rightarrow b\right)$ 
(15) $\left[\left(0 \rightarrow a^{\prime}\right) \rightarrow b\right]^{\prime}=(0 \rightarrow a) \rightarrow b^{\prime}$

(16) $\left(a^{\prime} \rightarrow b\right)^{\prime}=(0 \rightarrow a) \rightarrow b^{\prime}$

$(17) b^{\prime} \rightarrow[(b \rightarrow d) \rightarrow a] \sqsubseteq 0 \rightarrow b$.

Lemma 3.4 Let $\mathbf{A} \in \mathbf{I}_{2,0}$ and let $a, b, e \in A$ such that $\left(a \rightarrow b^{\prime}\right)^{\prime}=a$ and $\left(0 \rightarrow e^{\prime}\right) \rightarrow b=b$, and let $d \in A$ be arbitrary. Then

(a) $b \rightarrow d=(0 \rightarrow(d \rightarrow e)) \rightarrow(b \rightarrow d)$

(b) $(0 \rightarrow e) \rightarrow a^{\prime}=a^{\prime}$

(c) $\left(0 \rightarrow e^{\prime}\right) \rightarrow a=a$.

(d) $(0 \rightarrow e) \rightarrow[a \rightarrow(a \rightarrow d)]=a \rightarrow d$.

\section{Proof}

(回)

$$
\begin{array}{rlrl}
b \rightarrow d & =\left[\left(0 \rightarrow e^{\prime}\right) \rightarrow b\right] \rightarrow d & & \text { by hypothesis } \\
& =\left[\left\{d^{\prime} \rightarrow\left(0 \rightarrow e^{\prime}\right)\right\} \rightarrow(b \rightarrow d)^{\prime}\right] \rightarrow\left[\left\{\left(0 \rightarrow e^{\prime}\right) \rightarrow b\right\} \rightarrow d\right] & & \text { by Lemma 2.6 (27) } \\
\text { using } x=d, y=0 \rightarrow e^{\prime}, z=b & & \\
& =\left[\left\{d^{\prime} \rightarrow\left(0 \rightarrow e^{\prime}\right)\right\} \rightarrow(b \rightarrow d)^{\prime}\right] \rightarrow(b \rightarrow d) & & \text { by hypothesis } \\
& =\left[\left\{d^{\prime} \rightarrow\left(0 \rightarrow e^{\prime}\right)\right\} \rightarrow 0^{\prime}\right] \rightarrow(b \rightarrow d) & & \text { by Lemma 2.6 (1) } \\
& =\left[0 \rightarrow\left\{d^{\prime} \rightarrow\left(0 \rightarrow e^{\prime}\right)\right\}^{\prime}\right] \rightarrow(b \rightarrow d) & & \text { by Lemma 2.5 (a) } \\
& =\left[0 \rightarrow\left\{(0 \rightarrow d) \rightarrow\left(0 \rightarrow e^{\prime}\right)^{\prime}\right\}\right] \rightarrow(b \rightarrow d) & & \text { by Lemma 2.6 (18) } \\
& =[0 \rightarrow(d \rightarrow e)] \rightarrow(b \rightarrow d) & & \text { by Lemma 2.6 (17). }
\end{array}
$$

(b) Using Lemma 3.3 (3) (twice), and (国) with $d=a^{\prime}$, we obtain $\left[0 \rightarrow\left(a^{\prime} \rightarrow e\right)\right] \rightarrow a^{\prime}=[0 \rightarrow$ $\left.\left(a^{\prime} \rightarrow e\right)\right] \rightarrow\left(b \rightarrow a^{\prime}\right)=b \rightarrow a^{\prime}=a^{\prime}$. Hence,

$(3.4)\left[0 \rightarrow\left(a^{\prime} \rightarrow e\right)\right] \rightarrow a^{\prime}=a^{\prime}$.

Then,

$$
\begin{array}{rlrl}
(0 \rightarrow e) \rightarrow a^{\prime} & =\left[0 \rightarrow\left(a^{\prime} \rightarrow e\right)\right] \rightarrow a^{\prime} & & \text { by Lemma 2.6 (19) } \\
& =a^{\prime} & \text { by (3.4) }
\end{array}
$$

(IC)

$$
\begin{array}{rlrl}
\left(0 \rightarrow e^{\prime}\right) \rightarrow a & =\left[0 \rightarrow(0 \rightarrow e)^{\prime}\right] \rightarrow a & \text { by Lemma 2.6 (9) } \\
& =\left[(0 \rightarrow e) \rightarrow 0^{\prime}\right] \rightarrow a & \text { by Lemma 2.5 (a) } \\
& =\left[(0 \rightarrow e) \rightarrow a^{\prime}\right] \rightarrow a & \text { by Lemma 2.6 (1) } \\
& =a^{\prime} \rightarrow a & & \text { by (b) } \\
& =a & & \text { by Lemma } 2.4 \text { (d) })
\end{array}
$$


(d)

$$
\begin{aligned}
& a \rightarrow d=\left[\left(0 \rightarrow e^{\prime}\right) \rightarrow a\right] \rightarrow d \quad \text { by item (IC) } \\
& =\left[\left\{d^{\prime} \rightarrow\left(0 \rightarrow e^{\prime}\right)\right\} \rightarrow(a \rightarrow d)^{\prime}\right] \rightarrow\left[\left\{\left(0 \rightarrow e^{\prime}\right) \rightarrow a\right\} \rightarrow d\right] \text { by Lemma 2.6 (27) with } \\
& =\left[\left\{d^{\prime} \rightarrow\left(0 \rightarrow e^{\prime}\right)\right\} \rightarrow(a \rightarrow d)^{\prime}\right] \rightarrow(a \rightarrow d) \quad \text { by item (IC) } \\
& =\left[\left\{d^{\prime} \rightarrow\left(0 \rightarrow e^{\prime}\right)\right\} \rightarrow 0^{\prime}\right] \rightarrow(a \rightarrow d) \quad \text { by Lemma 2.6 (11) } \\
& =\left[0 \rightarrow\left\{d^{\prime} \rightarrow\left(0 \rightarrow e^{\prime}\right)\right\}^{\prime}\right] \rightarrow(a \rightarrow d) \quad \text { by Lemma 2.5 (国) } \\
& =\left[0 \rightarrow\left\{(0 \rightarrow d) \rightarrow\left(0 \rightarrow e^{\prime}\right)^{\prime}\right\}\right] \rightarrow(a \rightarrow d) \quad \text { by Lemma [2.6 (8) with } \\
& =[0 \rightarrow(d \rightarrow e)] \rightarrow(a \rightarrow d) \\
& x=d, y=e^{\prime}
\end{aligned}
$$

Thus,

$(3.5) a \rightarrow d=[0 \rightarrow(d \rightarrow e)] \rightarrow(a \rightarrow d)$.

Now,

$$
\begin{aligned}
&(0 \rightarrow e) \rightarrow[a \rightarrow(a \rightarrow d)]= {[0 \rightarrow[\{a \rightarrow(a \rightarrow d)\} \rightarrow e]] \rightarrow[a \rightarrow(a \rightarrow d)] } \\
& \text { by Lemma [2.6 (19) with } x=a \rightarrow(a \rightarrow d), y=e \\
&=[0 \rightarrow[\{a \rightarrow(a \rightarrow d)\} \rightarrow e]] \rightarrow[a \rightarrow\{a \rightarrow(a \rightarrow d)\}]
\end{aligned}
$$

by Lemma 2.6 (21)

$$
=a \rightarrow[a \rightarrow(a \rightarrow d)]
$$

by (3.5) replacing $d$ with $a \rightarrow(a \rightarrow d)$

$$
=a \rightarrow d
$$

by Lemma 2.6 (21).

Thus, (d) is proved and the proof of the lemma is complete.

Each of the next three lemmas prove a crucial step in the proof of transitivity of $\sqsubseteq$.

Lemma 3.5 Let $\mathbf{A} \in \mathbf{I}_{2,0}$ and let $a, b, c \in A$ such that $a \sqsubseteq b$ and $b \sqsubseteq c$. Let $d, e, f \in A$ be arbitrary. Then
(1) $\left(0 \rightarrow c^{\prime}\right) \rightarrow b=b$
(2) $(0 \rightarrow c) \rightarrow[a \rightarrow(a \rightarrow d)]=a \rightarrow d$
(3) $(0 \rightarrow c) \rightarrow(a \rightarrow d)=a \rightarrow d$
(4) $\left[0 \rightarrow\left((0 \rightarrow b) \rightarrow c^{\prime}\right)\right] \rightarrow b=b$
(5) $\left\{d^{\prime} \rightarrow\left[0 \rightarrow\left((0 \rightarrow b) \rightarrow c^{\prime}\right)\right]\right\} \rightarrow(b \rightarrow d)^{\prime}=(b \rightarrow d)^{\prime}$
(6) $(b \rightarrow d) \rightarrow[e \rightarrow(b \rightarrow d)]^{\prime}=\left[e \rightarrow 0^{\prime}\right] \rightarrow(b \rightarrow d)^{\prime}$
(7) $\left[b \rightarrow\left(a \rightarrow c^{\prime}\right)\right] \rightarrow a=a$
(8) $(0 \rightarrow b) \rightarrow(a \rightarrow d)=a \rightarrow d$ 
(9) $0 \rightarrow[b \rightarrow(a \rightarrow d)]=0 \rightarrow(a \rightarrow d)$

(10) $0 \rightarrow[\{b \rightarrow(a \rightarrow d)\} \rightarrow e]=0 \rightarrow[(a \rightarrow d) \rightarrow e]$

(11) $\left[0 \rightarrow\left(d^{\prime} \rightarrow c\right)\right] \rightarrow(0 \rightarrow b)^{\prime}=(0 \rightarrow d) \rightarrow(0 \rightarrow b)^{\prime}$

(12) $0 \rightarrow\left(a^{\prime} \rightarrow c\right) \sqsubseteq 0 \rightarrow b$

(13) $(0 \rightarrow a) \rightarrow(0 \rightarrow b)^{\prime}=(0 \rightarrow a)^{\prime}$

(14) $0 \rightarrow\left(a^{\prime} \rightarrow c\right)=0 \rightarrow a$

(15) $(d \rightarrow e) \rightarrow\left[\{b \rightarrow(a \rightarrow f)\}^{\prime} \rightarrow(0 \rightarrow a)^{\prime}\right]=(d \rightarrow e) \rightarrow\left[\left(a^{\prime} \rightarrow b\right) \rightarrow\left(f^{\prime} \rightarrow a^{\prime}\right)\right]$.

Proof By hypothesis, we have $\left(a \rightarrow b^{\prime}\right)^{\prime}=a$ and $\left(b \rightarrow c^{\prime}\right)^{\prime}=b$.

(1)

$$
\begin{array}{rlrl}
\left(0 \rightarrow c^{\prime}\right) \rightarrow b & =\left(c \rightarrow 0^{\prime}\right) \rightarrow b & & \text { by Lemma 2.5 (国) } \\
& =\left[\left(b^{\prime} \rightarrow c\right) \rightarrow\left(0^{\prime} \rightarrow b\right)^{\prime}\right]^{\prime} & & \text { by (I) } \\
& =\left[\left(b^{\prime} \rightarrow c\right) \rightarrow b^{\prime}\right]^{\prime} & & \text { by Lemmaa 2.4 (国) } \\
& =\left[(0 \rightarrow c) \rightarrow b^{\prime}\right]^{\prime} & & \text { by Lemma 2.6 (15) } \\
& =\left[\left(c^{\prime} \rightarrow 0^{\prime}\right) \rightarrow b^{\prime}\right]^{\prime} & & \text { by Lemma 2.5 (国) } \\
& =\left[\left(b^{\prime \prime} \rightarrow c^{\prime}\right) \rightarrow\left(0^{\prime} \rightarrow b^{\prime}\right)^{\prime}\right]^{\prime \prime} & & \text { from (I) } \\
& =\left(b^{\prime \prime} \rightarrow c^{\prime}\right) \rightarrow\left(0^{\prime} \rightarrow b^{\prime}\right)^{\prime} & \\
& =\left(b \rightarrow c^{\prime}\right) \rightarrow\left(0^{\prime} \rightarrow b^{\prime}\right)^{\prime} & & \\
& =\left(b \rightarrow c^{\prime}\right) \rightarrow b^{\prime \prime} & & \text { by Lemmaa 2.4 (国) } \\
& =\left(b \rightarrow c^{\prime}\right) \rightarrow b & & \\
& =b^{\prime} \rightarrow b & & \text { by hypothesis } \\
& =b & & \text { by Lemma 2.4 (d) } .
\end{array}
$$

(2) This is immmediate from (1) and Lemma 3.4 (dd) with $e=c$.

(3) Using Lemma 2.6 (21) and (22) we have that $(0 \rightarrow c) \rightarrow(a \rightarrow d)=(0 \rightarrow c) \rightarrow[a \rightarrow(a \rightarrow$ $d)]=a \rightarrow d$, implying (3).

(4)

$$
\begin{aligned}
{\left[0 \rightarrow\left((0 \rightarrow b) \rightarrow c^{\prime}\right)\right] \rightarrow b } & =\left\{\left(b^{\prime} \rightarrow 0\right) \rightarrow\left[\left((0 \rightarrow b) \rightarrow c^{\prime}\right) \rightarrow b\right]^{\prime}\right\}^{\prime} & & \text { by (I) } \\
& =\left\{b \rightarrow\left[\left((0 \rightarrow b) \rightarrow c^{\prime}\right) \rightarrow b\right]^{\prime}\right\}^{\prime} & & \\
& =\left\{b \rightarrow\left(c^{\prime} \rightarrow b\right)^{\prime}\right\}^{\prime} & & \text { by Lemma 2.6 (18) } \\
& =\left\{\left(b^{\prime} \rightarrow b\right) \rightarrow\left(c^{\prime} \rightarrow b\right)^{\prime}\right\}^{\prime} & & \text { by Lemma 2.4 (dd) } \\
& =\left(b \rightarrow c^{\prime}\right) \rightarrow b & & \text { by (I) } \\
& =\left(b \rightarrow c^{\prime}\right)^{\prime \prime} \rightarrow b & & \\
& =b^{\prime} \rightarrow b & & \text { by hypothesis } \\
& =b & & \text { by Lemma 2.4 (di). }
\end{aligned}
$$

$(5)$

$$
\begin{aligned}
\left\{d^{\prime} \rightarrow\left[0 \rightarrow\left((0 \rightarrow b) \rightarrow c^{\prime}\right)\right]\right\} \rightarrow(b \rightarrow d)^{\prime} & =\left\{\left[\left[0 \rightarrow\left((0 \rightarrow b) \rightarrow c^{\prime}\right)\right] \rightarrow b\right] \rightarrow d\right\}^{\prime} & \text { by (I) } \\
& =(b \rightarrow d)^{\prime} & \text { by (4) }
\end{aligned}
$$


(6)

$$
\begin{array}{rlrl}
(b \rightarrow d) \rightarrow[e \rightarrow(b \rightarrow d)]^{\prime} & =[e \rightarrow(b \rightarrow d)] \rightarrow(b \rightarrow d)^{\prime} & & \text { by Lemma 2.6 (14) with } \\
& =\left[e \rightarrow 0^{\prime}\right] \rightarrow(b \rightarrow d)^{\prime} & & x=e, y=b \rightarrow d \\
& \text { by Lemma 2.6 (11). }
\end{array}
$$

$(7)$

$$
\begin{aligned}
{\left[b \rightarrow\left(a \rightarrow c^{\prime}\right)\right] \rightarrow a } & =\left[\left(a^{\prime} \rightarrow b\right) \rightarrow\left\{\left(a \rightarrow c^{\prime}\right) \rightarrow a\right\}^{\prime}\right]^{\prime} & & \text { by (I) } \\
& =\left[\left(a^{\prime} \rightarrow b\right) \rightarrow\left\{\left(0 \rightarrow c^{\prime}\right) \rightarrow a\right\}^{\prime}\right]^{\prime} & & \text { by Lemma 2.6 (5) } \\
& =\left[\left(a^{\prime} \rightarrow b\right) \rightarrow a^{\prime}\right]^{\prime} & & \text { by (1) and Lemma 3.4 (Ic) } \\
& =\left[(0 \rightarrow b) \rightarrow a^{\prime}\right]^{\prime} & & \text { by Lemma 2.6 (15) } \\
& =(a \rightarrow 0) \rightarrow\left(b \rightarrow a^{\prime}\right)^{\prime} & & \text { by (I) } \\
& =a^{\prime} \rightarrow\left(b \rightarrow a^{\prime}\right)^{\prime} & & \\
& =a^{\prime} \rightarrow a^{\prime \prime} & & \text { by Lemma 3.3 (3) } \\
& =a^{\prime} \rightarrow a & & \\
& =a & & \text { by Lemma 2.4 (d) } .
\end{aligned}
$$

(8)

$$
\begin{aligned}
\left(0 \rightarrow b^{\prime}\right) \rightarrow b & =\left(0 \rightarrow 0^{\prime}\right) \rightarrow b & & \text { by Lemma 2.6 (1) } \\
& =\left(0^{\prime \prime} \rightarrow 0^{\prime}\right) \rightarrow b & & \\
& =0^{\prime} \rightarrow b & & \text { by Lemma 2.4 (d) } \\
& =b & & \text { by Lemma 2.4 (国). }
\end{aligned}
$$

Hence, by the hypothesis, together with Lemma 3.4 (d) , we obtain that $(0 \rightarrow b) \rightarrow\{a \rightarrow$ $(a \rightarrow d)\}=a \rightarrow d$. Hence, by Lemma 2.6 (21), we have $(0 \rightarrow b) \rightarrow(a \rightarrow d)=a \rightarrow d$.

(9)

$$
\begin{aligned}
0 \rightarrow(a \rightarrow d) & =0 \rightarrow[(0 \rightarrow b) \rightarrow(a \rightarrow d)] & & \text { by (8) } \\
& =b \rightarrow[0 \rightarrow(a \rightarrow d)] & & \text { by Lemma 2.6 (25) with } x=b, y=a \rightarrow d \\
& =0 \rightarrow[b \rightarrow(a \rightarrow d)] . & & \text { by Lemma 2.6 (13). }
\end{aligned}
$$

$(10)$

$$
\begin{aligned}
0 \rightarrow[\{b \rightarrow(a \rightarrow d)\} \rightarrow e] & =[b \rightarrow(a \rightarrow d)] \rightarrow(0 \rightarrow e) & & \text { by Lemma 2.6 (13) } \\
& =0 \rightarrow[[0 \rightarrow\{b \rightarrow(a \rightarrow d)\}] \rightarrow e] & & \text { by Lemma 2.6 (25) } \\
& =0 \rightarrow[\{0 \rightarrow(a \rightarrow d)\} \rightarrow e] & & \text { by (19) } \\
& =(a \rightarrow d) \rightarrow(0 \rightarrow e) & & \text { by Lemma 2.6 (25) } \\
& =0 \rightarrow[(a \rightarrow d) \rightarrow e] & & \text { by Lemma 2.6 (13). }
\end{aligned}
$$

$$
\begin{array}{rlrl}
{\left[0 \rightarrow\left(d^{\prime} \rightarrow c\right)\right] \rightarrow(0 \rightarrow b)^{\prime}} & =\left[0 \rightarrow\left(d^{\prime} \rightarrow c\right)\right] \rightarrow\left(b^{\prime} \rightarrow 0^{\prime}\right)^{\prime} & & \text { by Lemma 2.5 (国) } \\
& =\left[\left\{\left(d^{\prime} \rightarrow c\right) \rightarrow b^{\prime}\right\} \rightarrow 0^{\prime}\right]^{\prime} & & \text { by (I) } \\
& =\left[\left\{\left(b \rightarrow d^{\prime}\right) \rightarrow\left(c \rightarrow b^{\prime}\right)^{\prime}\right\}^{\prime} \rightarrow 0^{\prime}\right]^{\prime} & \text { by (I) } \\
& =\left[\left\{\left(b \rightarrow d^{\prime}\right) \rightarrow b^{\prime \prime}\right\}^{\prime} \rightarrow 0^{\prime}\right]^{\prime} & & \text { by Lemma 3.3 (3) } \\
& =\left[\left\{\left(b \rightarrow d^{\prime}\right) \rightarrow b\right\}^{\prime} \rightarrow 0^{\prime}\right]^{\prime} & & \\
& =\left[\left\{\left(0 \rightarrow d^{\prime}\right) \rightarrow b\right\}^{\prime} \rightarrow 0^{\prime}\right]^{\prime} & & \text { by Lemma 2.6 (5) } \\
& =\left[0 \rightarrow\left\{\left(0 \rightarrow d^{\prime}\right) \rightarrow b\right\}\right]^{\prime} & & \text { by Lemma 2.5 (国) } \\
& =\left[\left(0 \rightarrow d^{\prime}\right) \rightarrow(0 \rightarrow b)\right]^{\prime} & & \text { by Lemma 2.6 (13) } \\
& =\left[\left(d \rightarrow 0^{\prime}\right) \rightarrow(0 \rightarrow b)\right]^{\prime} & & \\
& =(0 \rightarrow d) \rightarrow(0 \rightarrow b)^{\prime} & & \text { by Lemma 2.6 (11). }
\end{array}
$$


$(12)$

$$
\begin{aligned}
0 \rightarrow\left(a^{\prime} \rightarrow c\right) & =0 \rightarrow\left[\left(a \rightarrow b^{\prime}\right)^{\prime \prime} \rightarrow c\right] & & \text { by hyphotesis } \\
& =0 \rightarrow\left[\left(a \rightarrow b^{\prime}\right) \rightarrow c\right] & & \\
& \sqsubseteq 0 \rightarrow\left(b^{\prime} \rightarrow c\right) & & \text { by Lemma 2.6 (29) } \\
& =0 \rightarrow b & & \text { by hyphotesis and Lemma 3.3 (44). }
\end{aligned}
$$

$$
\begin{array}{rlrl}
(0 \rightarrow a) \rightarrow(0 \rightarrow b)^{\prime} & =\left[a^{\prime} \rightarrow(0 \rightarrow b)\right]^{\prime} & \text { by Lemma 2.6 (8) } \\
& =\left[0 \rightarrow\left(a^{\prime} \rightarrow b\right)\right]^{\prime} & \text { by Lemma 2.6 (13) } \\
& =(0 \rightarrow a)^{\prime} . & & \text { by hyphotesis and Lemma 3.3 (44). }
\end{array}
$$

$$
\begin{aligned}
0 \rightarrow\left(a^{\prime} \rightarrow c\right) & =\left[\left\{0 \rightarrow\left(a^{\prime} \rightarrow c\right)\right\} \rightarrow(0 \rightarrow b)^{\prime}\right]^{\prime} & & \text { by (12) } \\
& =\left[(0 \rightarrow a) \rightarrow(0 \rightarrow b)^{\prime}\right]^{\prime} & & \text { by (11) with } d=a \\
& =(0 \rightarrow a)^{\prime \prime} & & \text { by (13) } \\
& =0 \rightarrow a . & &
\end{aligned}
$$

(15)

$$
\begin{aligned}
(d \rightarrow e) \rightarrow\left[\left(a^{\prime} \rightarrow b\right) \rightarrow\right. & \left.\left(f^{\prime} \rightarrow a^{\prime}\right)\right] \\
=(d \rightarrow e) \rightarrow & {\left[\left\{\left(0 \rightarrow a^{\prime}\right) \rightarrow b\right\} \rightarrow\left(f^{\prime} \rightarrow a^{\prime}\right)\right] } \\
& \text { by Lemma 3.3 (1) } \\
=(d \rightarrow e) \rightarrow & {\left[\left\{\left(0 \rightarrow a^{\prime}\right) \rightarrow b\right\} \rightarrow\left\{(f \rightarrow 0) \rightarrow a^{\prime}\right\}\right] } \\
=(d \rightarrow e) \rightarrow & {\left[\left\{\left(0 \rightarrow a^{\prime}\right) \rightarrow b\right\} \rightarrow\left\{(a \rightarrow f) \rightarrow\left(0 \rightarrow a^{\prime}\right)^{\prime}\right\}^{\prime}\right] } \\
& \text { by }(\mathrm{I}) \\
=(d \rightarrow e) \rightarrow & {\left[\{b \rightarrow(a \rightarrow f)\} \rightarrow\left(0 \rightarrow a^{\prime}\right)^{\prime}\right]^{\prime} } \\
& \quad \text { by }(\mathrm{I}) \\
=(d \rightarrow e) \rightarrow & {\left[\{b \rightarrow(a \rightarrow f)\}^{\prime} \rightarrow\left(a^{\prime} \rightarrow 0^{\prime}\right)^{\prime}\right] } \\
& \text { by (30) with } x=b \rightarrow(a \rightarrow f) \text { and } y=a^{\prime} \\
=(d \rightarrow e) \rightarrow & {\left[\{b \rightarrow(a \rightarrow f)\}^{\prime} \rightarrow(0 \rightarrow a)^{\prime}\right] } \\
& \quad \text { by Lemma } 2.5 \text { (国). }
\end{aligned}
$$

Hence, we have $(d \rightarrow e) \rightarrow\left[\{b \rightarrow(a \rightarrow f)\}^{\prime} \rightarrow(0 \rightarrow a)^{\prime}\right]=(d \rightarrow e) \rightarrow\left[\left(a^{\prime} \rightarrow b\right) \rightarrow\left(f^{\prime} \rightarrow\right.\right.$ $\left.\left.a^{\prime}\right)\right]$.

Lemma 3.6 Let $\mathbf{A} \in \mathbf{I}_{2,0}$ and let $a, b, c \in A$ such that $a \sqsubseteq b$ and $b \sqsubseteq c$. Let $d \in A$ be arbitrary.

Then
(a) $\left[c \rightarrow\left(b \rightarrow a^{\prime}\right)\right] \rightarrow b=\left(0 \rightarrow a^{\prime}\right) \rightarrow b$
(b) $\left(c \rightarrow a^{\prime}\right) \rightarrow b=a^{\prime} \rightarrow b$
(c) $\left(a^{\prime} \rightarrow b\right) \rightarrow\left(c \rightarrow a^{\prime}\right)=c \rightarrow a^{\prime}$
(d) $c \rightarrow a^{\prime}=a \rightarrow\left[b \rightarrow\left(a \rightarrow c^{\prime}\right)\right]$
(e) $0 \rightarrow(a \rightarrow d)=0 \rightarrow[c \rightarrow(a \rightarrow d)]$ 
(f) $(d \rightarrow a) \rightarrow d \sqsubseteq\left(a^{\prime} \rightarrow b\right) \rightarrow d$

(g) $\left(a^{\prime} \rightarrow b\right) \rightarrow c^{\prime}=(0 \rightarrow a) \rightarrow b^{\prime}$

(h) $0 \rightarrow\left(a \rightarrow c^{\prime}\right) \sqsubseteq 0 \rightarrow a^{\prime}$

(i) $0 \rightarrow\left(a \rightarrow c^{\prime}\right)=0 \rightarrow a^{\prime}$.

(j) $c \rightarrow\left(a \rightarrow c^{\prime}\right) \sqsubseteq 0 \rightarrow\left(a \rightarrow c^{\prime}\right)$

(k) $c \rightarrow\left(a \rightarrow c^{\prime}\right) \sqsubseteq 0 \rightarrow a^{\prime}$

(l) $\left(c \rightarrow\left(a \rightarrow c^{\prime}\right)\right)^{\prime} \rightarrow(0 \rightarrow a)^{\prime}=c \rightarrow\left(a \rightarrow c^{\prime}\right)$

(m) $a \rightarrow\left[b \rightarrow\left(a \rightarrow c^{\prime}\right)\right]=a \rightarrow c^{\prime}$

(n) $c \rightarrow a^{\prime}=a \rightarrow c^{\prime}$.

\section{Proof}

(a) Since $\left(b \rightarrow c^{\prime}\right)^{\prime}=b$, by Lemma 3.3 (10) with $d=a^{\prime}$, we have $\left(c \rightarrow\left(b \rightarrow a^{\prime}\right)\right) \rightarrow b=(0 \rightarrow$ $\left.a^{\prime}\right) \rightarrow b$.

(b)

$$
\begin{aligned}
\left(c \rightarrow a^{\prime}\right) \rightarrow b & =\left[c \rightarrow\left(b \rightarrow a^{\prime}\right)\right] \rightarrow b & & \text { by Lemma 3.3 (3) } \\
& =\left(0 \rightarrow a^{\prime}\right) \rightarrow b & & \text { by (回), }
\end{aligned}
$$

from which we get $\left(c \rightarrow a^{\prime}\right) \rightarrow b=\left(0 \rightarrow a^{\prime}\right) \rightarrow b$, which, together with Lemma 3.3 (1), implies $\left(c \rightarrow a^{\prime}\right) \rightarrow b=a^{\prime} \rightarrow b$.

(c)

$$
\begin{aligned}
c \rightarrow a^{\prime} & =(0 \rightarrow a) \rightarrow\left(c \rightarrow a^{\prime}\right) & & \text { by Lemma 2.6 (2) with } x=a^{\prime}, y=c \\
& =\left[0 \rightarrow\left(a^{\prime} \rightarrow b\right)\right] \rightarrow\left(c \rightarrow a^{\prime}\right) & & \text { by Lemma 3.3 (4) } \\
& =\left[0 \rightarrow\left\{\left(c \rightarrow a^{\prime}\right) \rightarrow b\right\}\right] \rightarrow\left(c \rightarrow a^{\prime}\right) & & \text { by (b) } \\
& =\left[\left(c \rightarrow a^{\prime}\right) \rightarrow(0 \rightarrow b)\right] \rightarrow\left(c \rightarrow a^{\prime}\right) & & \text { by Lemma 2.6 (13) } \\
& =[0 \rightarrow(0 \rightarrow b)] \rightarrow\left(c \rightarrow a^{\prime}\right) & & \text { by Lemma 2.6 (15) } \\
& =(0 \rightarrow b) \rightarrow\left(c \rightarrow a^{\prime}\right) & & \text { by Lemma 2.6 (6) } \\
& =\left[\left(c \rightarrow a^{\prime}\right) \rightarrow b\right] \rightarrow\left(c \rightarrow a^{\prime}\right) & & \text { by Lemma 2.6 (15) } \\
& =\left(a^{\prime} \rightarrow b\right) \rightarrow\left(c \rightarrow a^{\prime}\right) & & \text { by (b). }
\end{aligned}
$$


(d)

$$
\begin{aligned}
c \rightarrow a^{\prime}= & (0 \rightarrow a) \rightarrow\left(c \rightarrow a^{\prime}\right) \\
= & (0 \rightarrow a) \rightarrow\left[\left(a^{\prime} \rightarrow b\right) \rightarrow\left(c \rightarrow a^{\prime}\right)\right] \\
& =(0 \rightarrow a) \rightarrow\left[\left(a^{\prime} \rightarrow b\right) \rightarrow\left(c^{\prime \prime} \rightarrow a^{\prime}\right)\right] \\
& =(0 \rightarrow a) \rightarrow\left[\left\{b \rightarrow\left(a \rightarrow c^{\prime}\right)\right\}^{\prime} \rightarrow(0 \rightarrow a)^{\prime}\right] \\
& =(0 \rightarrow a) \rightarrow\left[\left\{b \rightarrow\left(a \rightarrow c^{\prime}\right)\right\}^{\prime} \rightarrow\left\{0 \rightarrow\left(a^{\prime} \rightarrow c\right)\right\}^{\prime}\right] \\
& =(0 \rightarrow a) \rightarrow\left[\left\{b \rightarrow\left(a \rightarrow c^{\prime}\right)\right\}^{\prime} \rightarrow\left\{0 \rightarrow\left(a \rightarrow c^{\prime}\right)^{\prime}\right\}^{\prime}\right] \\
& =(0 \rightarrow a) \rightarrow\left[\left\{b \rightarrow\left(a \rightarrow c^{\prime}\right)\right\}^{\prime} \rightarrow\left[0 \rightarrow\left\{b \rightarrow\left(a \rightarrow c^{\prime}\right)\right\}^{\prime}\right]^{\prime}\right] \\
& =\left[b \rightarrow\left(a \rightarrow c^{\prime}\right)\right]^{\prime} \rightarrow\left[(a \rightarrow 0) \rightarrow\left\{b \rightarrow\left(a \rightarrow c^{\prime}\right)\right\}^{\prime}\right]^{\prime} \\
& \\
& {\left[\{0 \rightarrow(a \rightarrow 0)\} \rightarrow\left\{b \rightarrow\left(a \rightarrow c^{\prime}\right)\right\}^{\prime}\right]^{\prime} } \\
= & {\left[\{a \rightarrow(0 \rightarrow 0)\} \rightarrow\left\{b \rightarrow\left(a \rightarrow c^{\prime}\right)\right\}^{\prime}\right]^{\prime} } \\
= & {\left[\left(a \rightarrow 0^{\prime}\right) \rightarrow\left\{b \rightarrow\left(a \rightarrow c^{\prime}\right)\right\}^{\prime}\right]^{\prime} } \\
= & {\left[\left\{b \rightarrow\left(a \rightarrow c^{\prime}\right)\right\} \rightarrow\left[a \rightarrow\left\{b \rightarrow\left(a \rightarrow c^{\prime}\right)\right\}\right]^{\prime}\right]^{\prime} } \\
& =\left[\left\{b \rightarrow\left(a \rightarrow c^{\prime}\right)\right\} \rightarrow a\right] \rightarrow\left[b \rightarrow\left(a \rightarrow c^{\prime}\right)\right] \\
= & a \rightarrow\left[b \rightarrow\left(a \rightarrow c^{\prime}\right)\right]
\end{aligned}
$$

by Lemma 2.6 (2)

by (c)

by Lemma 3.5 (15) with $d=0, e=a, f=c^{\prime}$ by Lemma 3.5 (14) by Lemma 2.6 (16) by Lemma 3.5(10) with $d=c^{\prime}, e=0$

by Lemma 2.6 (24) with $x=\left[b \rightarrow\left(a \rightarrow c^{\prime}\right)\right]^{\prime}$, $y=a, z=0$

by Lemma 2.6 (41) and (5) with $x=a \rightarrow 0$, $y=\left[b \rightarrow\left(a \rightarrow c^{\prime}\right)\right]^{\prime}$ by Lemma 2.6 (13) by Lemma 3.5)(6) with $e=a, d=a \rightarrow c^{\prime}$ by Lemma 2.6 (4) by Lemma 3.5(7).

(e)

$$
\begin{aligned}
0 \rightarrow(a \rightarrow d) & =0 \rightarrow[(0 \rightarrow c) \rightarrow(a \rightarrow d)] & & \text { by Lemma 3.5)(3) } \\
& =c \rightarrow[0 \rightarrow(a \rightarrow d)] & & \text { by Lemma 2.6 (25) } \\
& =0 \rightarrow[c \rightarrow(a \rightarrow d)] & & \text { by Lemma 2.6 (13). }
\end{aligned}
$$

(f)

$$
\begin{aligned}
(d \rightarrow a) \rightarrow d & =(0 \rightarrow a) \rightarrow d & & \text { by Lemma 2.6 (5) } \\
& =\left[0 \rightarrow\left(a^{\prime} \rightarrow b\right)\right] \rightarrow d & & \text { by Lemma 3.3 (41) } \\
& \sqsubseteq\left(a^{\prime} \rightarrow b\right) \rightarrow d & & \text { by Lemma 2.6 (36) }
\end{aligned}
$$

(g)

$$
\begin{aligned}
\left(a^{\prime} \rightarrow b\right) \rightarrow c^{\prime} & =\left[\left(c \rightarrow a^{\prime}\right) \rightarrow\left(b \rightarrow c^{\prime}\right)^{\prime}\right]^{\prime} & & \text { by (I) } \\
& =\left[\left(c \rightarrow a^{\prime}\right) \rightarrow b\right]^{\prime} & & \text { by hypothesis } \\
& =\left[\left\{c \rightarrow\left(b \rightarrow a^{\prime}\right)\right\} \rightarrow b\right]^{\prime} & & \text { by Lemma 3.3 (3) } \\
& =\left[\left(0 \rightarrow a^{\prime}\right) \rightarrow b\right]^{\prime} & & \text { by Lemma 3.3 (10) with } \\
& & & d=a^{\prime} \text { since } b \sqsubseteq c \\
& =\left[\left(b \rightarrow a^{\prime}\right) \rightarrow b\right]^{\prime} & & \text { by Lemma 2.6 (15) } \\
& =\left[\left(b \rightarrow a^{\prime}\right) \rightarrow b^{\prime \prime}\right]^{\prime} & & \\
& =\left[\left(b \rightarrow a^{\prime}\right) \rightarrow\left(0^{\prime} \rightarrow b^{\prime}\right)^{\prime}\right]^{\prime} & & \text { by Lemma 2.4 (国) } \\
& =\left(a^{\prime} \rightarrow 0^{\prime}\right) \rightarrow b^{\prime} & & \text { by (I) } \\
& =(0 \rightarrow a) \rightarrow b^{\prime} . & & \text { by Lemma 2.5 (国). }
\end{aligned}
$$

Hence, one has $\left(a^{\prime} \rightarrow b\right) \rightarrow c^{\prime}=(0 \rightarrow a) \rightarrow b^{\prime}$.

(h) From Lemma 3.5 (11), we have $\left(0 \rightarrow c^{\prime}\right) \rightarrow b=b$. Hence, we can use Lemma 3.3. Therefore, we have 


$$
\begin{aligned}
& 0 \rightarrow\left(a \rightarrow c^{\prime}\right)=0 \rightarrow\left[\left\{\left(0 \rightarrow c^{\prime}\right) \rightarrow a\right\} \rightarrow c^{\prime}\right] \text { by Lemma } 3.4 \text { (IC) and Lemma 3.5.(1) } \\
& =\left[\left(0 \rightarrow c^{\prime}\right) \rightarrow a\right] \rightarrow\left(0 \rightarrow c^{\prime}\right) \text { by Lemma 2.6 (13) } \\
& \sqsubseteq\left(a^{\prime} \rightarrow b\right) \rightarrow\left(0 \rightarrow c^{\prime}\right) \quad \text { by }(\mathbb{f}) \text { with } d=0 \rightarrow c^{\prime} \\
& =0 \rightarrow\left[\left(a^{\prime} \rightarrow b\right) \rightarrow c^{\prime}\right] \quad \text { by Lemma } 2.6 \text { (13) } \\
& =0 \rightarrow\left[(0 \rightarrow a) \rightarrow b^{\prime}\right] \quad \text { by }(\mathrm{g}) \\
& =0 \rightarrow\left[(b \rightarrow 0) \rightarrow\left(a \rightarrow b^{\prime}\right)^{\prime}\right]^{\prime} \text { by }(\mathrm{I}) \\
& =0 \rightarrow\left[b^{\prime} \rightarrow\left(a \rightarrow b^{\prime}\right)^{\prime}\right]^{\prime} \\
& =0 \rightarrow\left(b^{\prime} \rightarrow a\right)^{\prime} \quad \text { by hypothesis } \\
& =0 \rightarrow\left(b \rightarrow a^{\prime}\right) \quad \text { by Lemma } 2.6 \text { (16) } \\
& =0 \rightarrow a^{\prime} \quad \text { by Lemma } 3.3 \text { (3). }
\end{aligned}
$$

(i)

$$
\begin{aligned}
& 0 \rightarrow a^{\prime}=0 \rightarrow(a \rightarrow 0) \\
& =0 \rightarrow[c \rightarrow(a \rightarrow 0)] \quad \text { by (昰) } \\
& =0 \rightarrow\left(c \rightarrow a^{\prime}\right) \\
& =0 \rightarrow\left[\left(a \rightarrow c^{\prime}\right)^{\prime} \rightarrow(0 \rightarrow a)^{\prime}\right] \quad \text { by Lemma 2.6 (33) } \\
& =\left[0 \rightarrow\left(a \rightarrow c^{\prime}\right)\right]^{\prime} \rightarrow(0 \rightarrow a)^{\prime} \quad \text { by Lemma 2.6 (34) and Lemma 2.6 (6) } \\
& =\left[0 \rightarrow\left(a \rightarrow c^{\prime}\right)\right]^{\prime} \rightarrow\left(a^{\prime} \rightarrow 0^{\prime}\right)^{\prime} \quad \text { by Lemma } 2.5 \text { (a) } \\
& =\left[\left\{0 \rightarrow\left(a \rightarrow c^{\prime}\right)\right\} \rightarrow\left(0 \rightarrow a^{\prime}\right)^{\prime}\right]^{\prime} \text { by Lemma 2.5 (30) with } x=0 \rightarrow\left(a \rightarrow c^{\prime}\right), y=a^{\prime} \\
& =0 \rightarrow\left(a \rightarrow c^{\prime}\right) \quad \text { by (hi). }
\end{aligned}
$$

(j)

$$
\begin{aligned}
{\left[\left\{c \rightarrow\left(a \rightarrow c^{\prime}\right)\right\} \rightarrow\left\{0 \rightarrow\left(a \rightarrow c^{\prime}\right)\right\}^{\prime}\right]^{\prime} } & =\left[\left\{0 \rightarrow\left(a \rightarrow c^{\prime}\right)\right\} \rightarrow c\right] \rightarrow\left[\left(a \rightarrow c^{\prime}\right) \rightarrow\left\{0 \rightarrow\left(a \rightarrow c^{\prime}\right)\right\}^{\prime}\right]^{\prime} \\
& =\left[\left\{0 \rightarrow\left(a \rightarrow c^{\prime}\right)\right\} \rightarrow c\right] \rightarrow\left[\left\{\left(a \rightarrow c^{\prime}\right) \rightarrow 0\right\} \rightarrow\left(a \rightarrow c^{\prime}\right)\right] \\
& \text { by Lemma 2.6 (4) } \\
& =\left[\left\{0 \rightarrow\left(a \rightarrow c^{\prime}\right)\right\} \rightarrow c\right] \rightarrow\left[\left(a \rightarrow c^{\prime}\right)^{\prime} \rightarrow\left(a \rightarrow c^{\prime}\right)\right] \\
& =\left[\left\{0 \rightarrow\left(a \rightarrow c^{\prime}\right)\right\} \rightarrow c\right] \rightarrow\left(a \rightarrow c^{\prime}\right) \\
& =c \rightarrow\left(a \rightarrow c^{\prime}\right)
\end{aligned}
$$

by Lemma 2.6 (18) with

$$
x=a \rightarrow c^{\prime}, y=c \text {. }
$$

(k) From (ij) we have that $c \rightarrow\left(a \rightarrow c^{\prime}\right) \sqsubseteq 0 \rightarrow\left(a \rightarrow c^{\prime}\right)$. Then using (ii) we get $c \rightarrow\left(a \rightarrow c^{\prime}\right) \sqsubseteq$ $0 \rightarrow a^{\prime}$.

(l)

$$
\begin{aligned}
{\left[c \rightarrow\left(a \rightarrow c^{\prime}\right)\right]^{\prime} \rightarrow(0 \rightarrow a)^{\prime} } & =\left[c \rightarrow\left(a \rightarrow c^{\prime}\right)\right]^{\prime} \rightarrow\left(a^{\prime} \rightarrow 0^{\prime}\right)^{\prime} & & \text { by Lemma 2.5 (a) } \\
& =\left[\left\{c \rightarrow\left(a \rightarrow c^{\prime}\right)\right\} \rightarrow\left(0 \rightarrow a^{\prime}\right)^{\prime}\right]^{\prime} & & \text { by Lemma 2.6 (30) } \\
& =c \rightarrow\left(a \rightarrow c^{\prime}\right) & & \text { by (a) . }
\end{aligned}
$$

(m)

$$
\begin{aligned}
a \rightarrow\left[b \rightarrow\left(a \rightarrow c^{\prime}\right)\right]= & =c \rightarrow a^{\prime} & & \text { by (dd) } \\
& =c^{\prime \prime} \rightarrow a^{\prime} & & \\
& =\left(a \rightarrow c^{\prime}\right)^{\prime} \rightarrow(0 \rightarrow a)^{\prime} & & \text { by Lemma 2.6 (33) } \\
& =\left[c \rightarrow\left(a \rightarrow c^{\prime}\right)\right]^{\prime} \rightarrow(0 \rightarrow a)^{\prime} & & \text { by Lemma 2.6 (17) } \\
& =c \rightarrow\left(a \rightarrow c^{\prime}\right) & & \text { by (1) } \\
& =a \rightarrow c^{\prime} & & \text { by Lemma 2.6 (17). }
\end{aligned}
$$


(n) From (dd) and (m), we get $c \rightarrow a^{\prime}=a \rightarrow c^{\prime}$.

Lemma 3.7 Let $\mathbf{A} \in \mathbf{I}_{2,0}$ and let $a, b, c \in A$ such that $a \sqsubseteq b$ and $b \sqsubseteq c$. Then
(a) $c^{\prime} \rightarrow[(c \rightarrow d) \rightarrow b] \sqsubseteq c$
(b) $0 \rightarrow a^{\prime}=c \rightarrow\left(0 \rightarrow a^{\prime}\right)$
(c) $c^{\prime} \rightarrow\left(a^{\prime} \rightarrow b\right) \sqsubseteq c$
(d) $\left(0 \rightarrow a^{\prime}\right) \rightarrow b=\left(c \rightarrow a^{\prime}\right) \rightarrow b$
(e) $c^{\prime} \rightarrow\left(a^{\prime} \rightarrow b\right) \sqsubseteq 0 \rightarrow c$
(f) $\left[(0 \rightarrow a) \rightarrow b^{\prime}\right] \rightarrow c=c^{\prime} \rightarrow\left(a^{\prime} \rightarrow b\right)$
(g) $a^{\prime} \rightarrow c=c^{\prime} \rightarrow\left(a^{\prime} \rightarrow b\right)$
(h) $a^{\prime} \rightarrow c \sqsubseteq c$
(i) $a^{\prime} \rightarrow c=\left(0 \rightarrow a^{\prime}\right) \rightarrow c$.

\section{Proof}

(a)

$$
\begin{array}{rlrl}
c^{\prime} \rightarrow[(c \rightarrow d) \rightarrow b] & =c^{\prime} \rightarrow\left[(c \rightarrow d) \rightarrow\left(b \rightarrow c^{\prime}\right)^{\prime}\right] & & \text { by hypothesis } \\
& =c^{\prime} \rightarrow\left[(d \rightarrow b) \rightarrow c^{\prime}\right]^{\prime} & & \text { by (I) } \\
& \sqsubseteq c^{\prime \prime} & & \text { by Lemma 2.6 (37) } \\
& =c .
\end{array}
$$

(b)

$$
\begin{array}{rlrl}
0 \rightarrow a^{\prime} & =b \rightarrow\left(0 \rightarrow a^{\prime}\right) & & \text { by Lemma 3.3 (11) } \\
& =\left[0 \rightarrow\left\{\left(0 \rightarrow a^{\prime}\right) \rightarrow c\right\}\right] \rightarrow\left[b \rightarrow\left(0 \rightarrow a^{\prime}\right)\right] & \text { by Lemma 3.5 (11) } \\
& & \text { and Lemma 3.4 (国) } \\
& =\left[0 \rightarrow\left\{\left(0 \rightarrow a^{\prime}\right) \rightarrow c\right\}\right] \rightarrow\left(0 \rightarrow a^{\prime}\right) & & \text { with } d=0 \rightarrow a^{\prime}, e=c \\
& =\left[\left(0 \rightarrow a^{\prime}\right) \rightarrow(0 \rightarrow c)\right] \rightarrow\left(0 \rightarrow a^{\prime}\right) & & \text { by Lemma Lemma 2.3 (11) } \\
& =[0 \rightarrow(0 \rightarrow c)] \rightarrow\left(0 \rightarrow a^{\prime}\right) & & \text { by Lemma 2.6 (15) } \\
& =(0 \rightarrow c) \rightarrow\left(0 \rightarrow a^{\prime}\right) & & \text { by Lemma 2.6 (21) } \\
& =c \rightarrow\left(0 \rightarrow a^{\prime}\right) & & \text { by Lemma 2.6 (20). }
\end{array}
$$

(c)

$$
\begin{aligned}
& c^{\prime} \rightarrow\left(a^{\prime} \rightarrow b\right)=c^{\prime} \rightarrow\left[\left(0 \rightarrow a^{\prime}\right) \rightarrow b\right] \quad \text { by Lemma } 3.3 \text { (1) } \\
& =c^{\prime} \rightarrow\left[\left\{c \rightarrow\left(0 \rightarrow a^{\prime}\right)\right\} \rightarrow b\right] \text { by (b) } \\
& \sqsubseteq c \quad \text { by (回) with } d=0 \rightarrow a^{\prime} \text {. }
\end{aligned}
$$


(d)

$$
\begin{aligned}
\left(0 \rightarrow a^{\prime}\right) \rightarrow b & =\left[c \rightarrow\left(0 \rightarrow a^{\prime}\right)\right] \rightarrow b & & \text { by (b) } \\
& =\left[\left(b^{\prime} \rightarrow c\right) \rightarrow\left\{\left(0 \rightarrow a^{\prime}\right) \rightarrow b\right\}^{\prime}\right]^{\prime} & & \text { by (I) } \\
& =\left[\left(b^{\prime} \rightarrow c\right) \rightarrow\left\{\left(b^{\prime} \rightarrow 0\right) \rightarrow\left(a^{\prime} \rightarrow b\right)^{\prime}\right\}\right]^{\prime} & & \text { by (I) and } x^{\prime \prime} \approx x \\
& =\left[\left(b^{\prime} \rightarrow c\right) \rightarrow\left\{b \rightarrow\left(a^{\prime} \rightarrow b\right)^{\prime}\right\}\right]^{\prime} & & \\
& =\left[\left(b^{\prime} \rightarrow c\right) \rightarrow\left\{\left(a^{\prime} \rightarrow 0^{\prime}\right) \rightarrow b^{\prime}\right\}\right]^{\prime} & & \text { by Lemma 2.6 (26) } \\
& =\left[\left(b^{\prime} \rightarrow c\right) \rightarrow\left\{(0 \rightarrow a) \rightarrow b^{\prime}\right\}\right]^{\prime} & & \text { by Lemma 2.5 (a) } \\
& =\left[\left(b^{\prime} \rightarrow c\right) \rightarrow\left(a^{\prime} \rightarrow b\right)^{\prime}\right]^{\prime} & & \text { by Lemma 3.3 (16) } \\
& =\left(c \rightarrow a^{\prime}\right) \rightarrow b & & \text { by (I). }
\end{aligned}
$$

(e)

$$
\begin{aligned}
c^{\prime} \rightarrow\left(a^{\prime} \rightarrow b\right) & =c^{\prime} \rightarrow\left[\left(0 \rightarrow a^{\prime}\right) \rightarrow b\right] & \text { by Lemma } 3.3 \text { (15) and Lemma } 3.3 \text { (16) } \\
& =c^{\prime} \rightarrow\left[\left(c \rightarrow a^{\prime}\right) \rightarrow b\right] & \text { by (dd) } \\
& \sqsubseteq 0 \rightarrow c . & \text { by Lemma } 3.3 \text { (17) with } d=a^{\prime} .
\end{aligned}
$$

(f)

$$
\begin{aligned}
c^{\prime} \rightarrow\left(a^{\prime} \rightarrow b\right) & =\left[\left\{c^{\prime} \rightarrow\left(a^{\prime} \rightarrow b\right)\right\} \rightarrow(0 \rightarrow c)^{\prime}\right]^{\prime} & & \text { by (ㅌ) } \\
& =\left[\left(a^{\prime} \rightarrow b\right) \rightarrow 0\right] \rightarrow c & & \text { by (I) } \\
& =\left(a^{\prime} \rightarrow b\right)^{\prime} \rightarrow c & & \\
& =\left[(0 \rightarrow a) \rightarrow b^{\prime}\right] \rightarrow c & & \text { by Lemma } 3.3 \text { (16). }
\end{aligned}
$$

(g)

$$
\begin{aligned}
c^{\prime} \rightarrow\left(a^{\prime} \rightarrow b\right) & =\left((0 \rightarrow a) \rightarrow b^{\prime}\right) \rightarrow c & & \text { by (ff) } \\
& =\left[(0 \rightarrow a) \rightarrow 0^{\prime}\right] \rightarrow\left(b^{\prime} \rightarrow c\right) & & \text { by Lemma 3.3 (14) with } d=0 \rightarrow a \\
& =\left[\left(a^{\prime} \rightarrow 0^{\prime}\right) \rightarrow 0^{\prime}\right] \rightarrow\left(b^{\prime} \rightarrow c\right) & & \text { by Lemma 2.5 (国) } \\
& =\left[\left(a^{\prime} \rightarrow 0\right) \rightarrow 0^{\prime}\right] \rightarrow\left(b^{\prime} \rightarrow c\right) & & \text { by Lemma 2.6 (11) } \\
& =\left[a^{\prime \prime} \rightarrow 0^{\prime}\right] \rightarrow\left(b^{\prime} \rightarrow c\right) & & \\
& =\left(a \rightarrow 0^{\prime}\right) \rightarrow\left(b^{\prime} \rightarrow c\right) & & \\
& =\left(a \rightarrow b^{\prime}\right) \rightarrow c & & \text { by Lemma 3.3 (14) with } d=a \\
& =a^{\prime} \rightarrow c & & \text { by hypothesis. }
\end{aligned}
$$

(h) This is immediate from (g) and (c).

(i)

$$
\begin{aligned}
& \left(0 \rightarrow a^{\prime}\right) \rightarrow c=\left(c \rightarrow a^{\prime}\right) \rightarrow c \quad \text { by Lemma 2.6 (5) } \\
& =\left[c \rightarrow\left(a^{\prime} \rightarrow c\right)^{\prime}\right]^{\prime} \text { by Lemma 2.6 (4) } \\
& =\left[\left(a^{\prime} \rightarrow c\right) \rightarrow c^{\prime}\right]^{\prime} \text { by Lemma 2.6 (14) } \\
& =a^{\prime} \rightarrow c \quad \text { by (h). }
\end{aligned}
$$

We are now ready to complete the proof of transitivity of $\sqsubseteq$.

Theorem $3.8 \sqsubseteq$ is transitive. 
Proof Let $a, b, c \in A$ such that $a \sqsubseteq b$ and $b \sqsubseteq c$. Observe that

$$
\begin{array}{rlrl}
a^{\prime} & =a \rightarrow 0 & & \\
& =(0 \rightarrow c) \rightarrow(a \rightarrow 0) & & \text { by Lemma } 3.5 \text { (13) with } d=0 \\
& =(0 \rightarrow c) \rightarrow a^{\prime} & & \\
& =\left(a^{\prime} \rightarrow c\right) \rightarrow a^{\prime} & & \text { by Lemma 2.6 (15) } \\
& =\left(\left(0 \rightarrow a^{\prime}\right) \rightarrow c\right) \rightarrow a^{\prime} & \text { by Lemma } 3.7 \text { (1i) } \\
& =c \rightarrow a^{\prime} & & \text { by Lemma 2.6 (18) } \\
& =a \rightarrow c^{\prime} & & \text { by Lemma } 3.6 \text { (10). }
\end{array}
$$

Consequently,

$$
a=a^{\prime \prime}=\left(a \rightarrow c^{\prime}\right)^{\prime},
$$

implying $a \sqsubseteq c$. Hence, $\sqsubseteq$ is transitive on $\mathbf{A}$.

We are now prepared to present our first main theorem.

Theorem 3.9 The variety $\mathbf{I}_{2,0}$ is a maximal subvariety of $\mathbf{I}$ with respect to the property that the relation $\sqsubseteq$ introduced in Definition 3.1 is a partial order.

Proof Let $\mathbf{A} \in \mathbf{I}_{2,0}$. The relation $\sqsubseteq$ is a partial order on $A$ in view of Lemma 2.4 (C), Lemma 3.2, and Theorem 3.8.

Next, let $\mathbf{V}$ be a subvariety of $\mathbf{I}$ such that $\sqsubseteq$ is a partial order on every algebra in $\mathbf{V}$. Now let $\mathbf{A} \in \mathbf{V}$. Reflexivity of $\sqsubseteq$ implies that $\mathbf{A} \models\left(x \rightarrow x^{\prime}\right)^{\prime} \approx x$. Therefore, by Lemma 2.4, we conclude that $\mathbf{A} \in \mathbf{I}_{2,0}$, and hence, $\mathbf{V} \subseteq \mathbf{I}_{2,0}$, completing the proof.

\section{A method to construct finite $\mathbf{I}_{2,0}$-chains}

Now that we know the relation $\sqsubseteq$ is a partial order on algebras in $\mathbf{I}_{2,0}$, it is natural to consider those algebras in $\mathbf{I}_{2,0}$, in which $\sqsubseteq$ is a total order.

Definition 4.1 Let $\mathbf{A} \in \mathbf{I}$. We say that $\mathbf{A}$ is an $\mathbf{I}_{2,0}$-chain (chain, for short) if $\mathbf{A} \in \mathbf{I}_{2,0}$ and the relation $\sqsubseteq$ (see Definition 3.1) is totally ordered on $A$.

In this section we describe a method of constructing finite $\mathbf{I}_{2,0}$-chains. But, first, we will present some examples of $\mathbf{I}_{2,0}$-chains that will foreshadow the method to construct finite $\mathbf{I}_{2,0}$-chains. We note that, in these examples, the number 0 is the constant element.

It is easy to see that the only 2 -element $\mathbf{I}_{2,0}$-chains, up to isomorphism, are

$$
\begin{array}{r|ll}
\rightarrow: & 0 & 1 \\
\hline 0 & 1 & 1 \\
1 & 0 & 1
\end{array} \quad \text { with } 0 \sqsubset 1 .
$$$$
\begin{array}{r|rr}
\rightarrow: & -1 & 0 \\
\hline-1 & -1 & -1 \\
0 & -1 & 0
\end{array} \quad \text { with }-1 \sqsubset 0
$$

and the only 3-element $\mathbf{I}_{2,0}$-chains, up to isomorphism, are

\begin{tabular}{r|lll}
$\rightarrow:$ & 0 & 1 & 2 \\
\hline 0 & 2 & 2 & 2 \\
1 & 1 & 1 & 2 \\
2 & 0 & 1 & 2
\end{tabular}$\quad$ with $0 \sqsubset 1 \sqsubset 2$,

\begin{tabular}{r|rrr}
$\rightarrow:$ & -1 & 0 & 1 \\
\hline-1 & -1 & -1 & -1 \\
0 & -1 & 1 & 1 \\
1 & -1 & 0 & 1
\end{tabular} with $-1 \sqsubset 0 \sqsubset 1$,




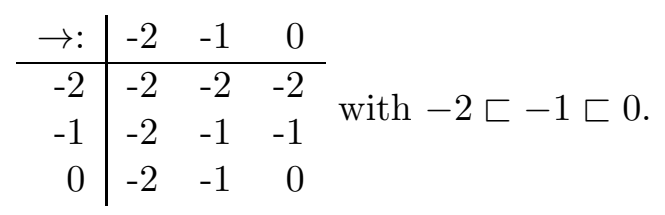

Note that, henceforth, we will use the symbol $\leq$ to denote the natural order in $\mathbb{Z}$. Recall that $\sqsubseteq$ is being used for the order (see Definition 3.1).

The next definition describes a general method to construct a class of finite $\mathbf{I}_{\mathbf{2}, \mathbf{0}^{-} \text {-chains, }}$ generalizing the above examples. In the next section, we will show that, this method, in fact, yields, up to isomorphism, all finite $\mathbf{I}_{\mathbf{2}, \mathbf{0}}$-chains.

Definition 4.2 Let $k \in \mathbb{N}$. Let $m, n \in \omega$ be such that the interval $[-n, m] \subseteq \mathbb{Z}$ with $|[-n, m]|=k$ and $0 \leq n, m \leq k-1$. The (auxiiliary) functions $p$ (predecessor) and $*$ are defined on $[-n, m]$ as follows:

$$
p(x)= \begin{cases}x-1 & \text { if } x>-n \\ -n & \text { if } x=-n\end{cases}
$$

and

$$
x^{*}= \begin{cases}m & \text { if } x=0 \\ x & \text { if } x<0 \\ p\left((p(x))^{*}\right) & \text { if } x>0 .\end{cases}
$$

For convenience, we write $p\left(p(x)^{*}\right)$ for $p\left((p(x))^{*}\right)$. (Notice that the function $*$ is defined recursively for $x \geq 0$.)

Define the algebra $[-\mathbf{n}, \mathbf{m}]$ as follows:

$$
\begin{gathered}
{[-\mathbf{n}, \mathbf{m}]:=\langle[-n, m] ; \Rightarrow, 0\rangle, \text { where } 0 \in[-n, m] \text { is the constant and } \Rightarrow \text { is defined by }} \\
x \Rightarrow y= \begin{cases}\max \left(x^{*}, y\right) & \text { if } x, y \geq 0 \\
\min (x, y) & \text { otherwise. }\end{cases}
\end{gathered}
$$

We set $x^{\prime}:=x \Rightarrow 0$.

We shall now illustrate the method described in the above definition by applying it to construct a 6-element $\mathbf{I}_{\mathbf{2}, \mathbf{0}}$-chain.

Let $k=6$, and consider the interval $A=[-2,3]=\{-2,-1,0,1,2,3\}$. Since $0 \Rightarrow 0=$ $\max \left(0^{*}, 0\right)=\max (3,0)=3$ and $a \Rightarrow b=\min (a, b)$ if $a<0$ or $b<0$, we arrive at tha following partial table for $\Rightarrow$ :

\begin{tabular}{c|cccccc}
\hline$\Rightarrow$ & -2 & -1 & 0 & 1 & 2 & 3 \\
\hline-2 & -2 & -2 & -2 & -2 & -2 & -2 \\
-1 & -2 & -1 & -1 & -1 & -1 & -1 \\
0 & -2 & -1 & 3 & $?$ & $?$ & $?$ \\
1 & -2 & -1 & $?$ & $?$ & $?$ & $?$ \\
2 & -2 & -1 & $?$ & $?$ & $?$ & $?$ \\
3 & -2 & -1 & $?$ & $?$ & $?$ & $?$
\end{tabular}

Next, we determine the operations $p$ and $*$ : 


\begin{tabular}{l|l}
\hline$x$ & $x^{*}$ \\
\hline 0 & 3 \\
1 & $p\left(p(1)^{*}\right)=p\left(0^{*}\right)=p(3)=2$ \\
2 & $p\left(p(2)^{*}\right)=p\left(1^{*}\right)=p(2)=1$ \\
3 & $p\left(p(3)^{*}\right)=p\left(2^{*}\right)=p(1)=0$
\end{tabular}

Hence the table for $\Rightarrow$ becomes:

\begin{tabular}{c|cccccc}
\hline$\Rightarrow$ & -2 & -1 & 0 & 1 & 2 & 3 \\
\hline-2 & -2 & -2 & -2 & -2 & -2 & -2 \\
-1 & -2 & -1 & -1 & -1 & -1 & -1 \\
0 & -2 & -1 & 3 & $?$ & $?$ & $?$ \\
1 & -2 & -1 & 2 & $?$ & $?$ & $?$ \\
2 & -2 & -1 & 1 & $?$ & $?$ & $?$ \\
3 & -2 & -1 & 0 & $?$ & $?$ & $?$
\end{tabular}

Observe that $0 \Rightarrow 1=\max \left(0^{*}, 1\right)=\max (3,1)=3,1 \Rightarrow 1=\max \left(1^{*}, 1\right)=\max (2,1)=2$, $2 \Rightarrow 1=\max \left(2^{*}, 1\right)=\max (1,1)=1$ and $3 \Rightarrow 1=\max \left(3^{*}, 1\right)=\max (0,1)=1$. Then we get

\begin{tabular}{|c|c|c|c|c|c|c|}
\hline$\Rightarrow$ & -2 & -1 & 0 & 1 & 2 & 3 \\
-2 & -2 & -2 & -2 & -2 & -2 & -2 \\
-1 & -2 & -1 & -1 & -1 & -1 & -1 \\
0 & -2 & -1 & 3 & 3 & $?$ & $?$ \\
1 & -2 & -1 & 2 & 2 & $?$ & $?$ \\
2 & -2 & -1 & 1 & 1 & $?$ & $?$ \\
3 & -2 & -1 & 0 & 1 & $?$ & $?$ \\
\hline
\end{tabular}

Iterating this process we obtain the following complete table for $\Rightarrow$ :

\begin{tabular}{|c|c|c|c|c|c|c|}
\hline$\Rightarrow$ & -2 & -1 & 0 & 1 & 2 & 3 \\
-2 & -2 & -2 & -2 & -2 & -2 & -2 \\
-1 & -2 & -1 & -1 & -1 & -1 & -1 \\
0 & -2 & -1 & 3 & 3 & 3 & 3 \\
1 & -2 & -1 & 2 & 2 & 2 & 3 \\
2 & -2 & -1 & 1 & 1 & 2 & 3 \\
3 & -2 & -1 & 0 & 1 & 2 & 3 \\
\hline
\end{tabular}

Thus we have constructed the algebra $[-\mathbf{n}, \mathbf{m}]$. Observe that $-2 \sqsubset-1 \sqsubset 0 \sqsubset 1 \sqsubset 2 \sqsubset 3$ and $x^{\prime \prime}=x^{* *}=x$. Also, it is routine to verify $[-\mathbf{n}, \mathbf{m}] \in \mathbf{I}_{\mathbf{2}, \mathbf{0}}$. Hence it is an $\mathbf{I}_{2,0}$-chain.

Returning to the general method, we now aim to prove that $[-\mathbf{n} ; \mathbf{m}]$ is an $\mathbf{I}_{2,0}$-chain. To prove this, we will need the following lemmas.

Lemma 4.3 If $x \in[-\mathbf{n}, \mathbf{m}]$ and $0 \leq x \leq m$ then $x^{*}=m-x$ and, consequently, $x^{*} \in[0, m]$.

Proof We prove this lemma by induction on the element $x$. Assume that $x=0$. Then $0^{*}=m=m-0$.

Next, suppose $x>0$. Since $-n \leq 0<x$, we have $p(x)=x-1$. Hence, by inductive hypothesis, we have

(4.1) $p(x)^{*}=m-p(x)=m-(x-1)=m-x+1$. 
From $x>0$, we can conclude that $m-x+1 \leq m$. Also, since $x \leq m$, we obtain $0 \leq m-x$, thus $-n-1<0 \leq m-x$, implying $m-x+1>-n$. Then we get $p(m-x+1)=m-x+1-1$. By (4.1), $x^{*}=p\left((p(x))^{*}\right)=p(m-x+1)=m-x$, completing the induction. It is clear that $x^{*} \in[0, m]$.

Corollary 4.4 If $x \in[-\mathbf{n}, \mathbf{m}]$ then $x^{\prime}=x^{*}$.

Proof If $x<0$ we have that $x^{\prime}=x \Rightarrow 0=\min (x, 0)=x=x^{*}$. If $x>0$, then by Lemma 4.3, $x^{*} \geq 0$, and hence $x^{\prime}=x \Rightarrow 0=\max \left(x^{*}, 0\right)=x^{*}$.

Lemma 4.5 If $x \in[-\mathbf{n}, \mathbf{m}]$ then $x^{\prime \prime}=x$.

Proof We consider the following cases:

- If $x<0$, then $x^{*}=x$, and hence $x^{* *}=x$.

- If $x \geq 0$,

$$
\begin{aligned}
& x^{* *}=(m-x)^{*} \quad \text { by Lemma } 4.3 \text { since } 0<x \leq m \\
& =m-(m-x) \text { by Lemma } 4.3 \text { since } 0 \leq m-x \leq m \\
& =x \text {. }
\end{aligned}
$$

Consequently, by Corollary 4.4, $x^{\prime \prime}=x$.

Lemma 4.6 If $x, y \in[-\mathbf{n}, \mathbf{m}]$ and $0 \leq x \leq y$ then $x^{*} \geq y^{*}$.

Proof We prove this lemma by induction on the element $x$. If $x=0, x^{*}=0^{*}=m \geq y^{*}$ by Lemma 4.3 .

Now assume that $x>0$. Since $0<x \leq y$, we have that $x^{*}=p\left(p(x)^{*}\right)$ and $y^{*}=p\left(p(y)^{*}\right)$. Note that $0 \leq p(x) \leq p(y)$. Then, by induction hypothesis, we get $p(y)^{*} \leq p(x)^{*}$. Hence $x^{*}=p\left(p(x)^{*}\right) \geq p\left(p(y)^{*}\right)=y^{*}$.

Lemma 4.7 Let $k \in \mathbb{N}$. Let $m, n \in \omega$ be such that the interval $[-n, m] \subseteq \mathbb{Z}$ with $|[-n, m]|=k$ and $0 \leq n, m \leq k-1$. Then, $[-\mathbf{n}, \mathbf{m}] \in \mathbf{I}_{2,0}$.

Proof The proof that $\langle[-n ; m] ; \Rightarrow, 0\rangle$ satisfies the identity (I) is long and computational, but routine. Hence we leave the verification to the reader with the recommendation that the following cases be considered, where $i, j, k \in[-n ; m]$ :
(1) $i, j, k \geq 0, i^{*} \geq j, i \geq k$
(7) $i \geq 0, j<0$ and $k \geq 0$
(2) $i, j, k \geq 0, i^{*} \geq j, i<k$
(8) $i \geq 0, j<0$ and $k<0$
(3) $i, j, k \geq 0, i^{*}<j, k \geq i$
(9) $i<0, j \geq 0$ and $k \geq 0$
(4) $i, j, k \geq 0, i^{*}<j, k<i, j^{*} \leq k$
(10) $i<0, j \geq 0$ and $k<0$
(5) $i, j, k \geq 0, i^{*}<j, k<i, j^{*}>k$
(11) $i<0, j<0$ and $k \geq 0$
(6) $i, j \geq 0$ and $k<0$
(12) $i, j, k<0$.

Observe that, if $x \in[-n, m]$, then, from Corollary 4.4, we have $x^{\prime}=x^{*}$, and from Lemma 4.5 we have that $x^{\prime \prime}=x$; and in particular $0^{\prime \prime}=0$. Thus, we conclude that $\langle[-n, m] ; \Rightarrow, 0\rangle \in \mathbf{I}_{2,0}$. 
In view of the above lemma and Theorem 3.8 , the relation defined by

$$
x \sqsubseteq y \quad \text { if and only if } \quad\left(x \Rightarrow y^{\prime}\right)^{\prime}=x
$$

is a partial order on $[-\mathbf{n}, \mathbf{m}]$. We now wish to show that $\sqsubseteq$ is indeed a total order.

Lemma 4.8 Let $[-\mathbf{n}, \mathbf{m}]$ be the algebra, as defined in Definition 4.2. Then

$$
\langle[-n, m] ; \sqsubseteq\rangle \cong\langle[-n, m] ; \leq\rangle \text {. }
$$

Proof Let $x, y \in[-n, m]$. It is enough to prove that $x \leq y$ if and only if $x \sqsubseteq y$.

Assume that $x \leq y$. We will consider the following cases:

- Case 1: $x<0$. Then

(4.2) $\left(x \Rightarrow y^{\prime}\right)^{\prime}=\left(x \Rightarrow y^{*}\right)^{*}=\left[\min \left(x, y^{*}\right)\right]^{*}$.

We consider further the following subcases:

- Case 1.1: $y<0$.

$$
\begin{aligned}
\left(x \Rightarrow y^{\prime}\right)^{\prime} & =\left[\min \left(x, y^{*}\right)\right]^{*} & & \text { by }(4.2) \\
& =[\min (x, y)]^{*} & & \text { since } y<0 \\
& =x^{*} & & \text { since } x \leq y \\
& =x . & & \text { since } x<0
\end{aligned}
$$

- Case 1.2: $y \geq 0$.

$$
\begin{aligned}
\left(x \Rightarrow y^{\prime}\right)^{\prime} & =\left[\min \left(x, y^{*}\right)\right]^{*} & & \text { by (4.2) } \\
& =x^{*} & & \text { since } y^{*} \geq 0 \text { by Lemma 4.3, and } x<0 \\
& =x . & &
\end{aligned}
$$

- Case 2: $x \geq 0$. Therefore $y \geq 0$. In this case

$$
\begin{array}{rlr}
\left(x \Rightarrow y^{\prime}\right)^{\prime} & =\left(x \Rightarrow y^{*}\right)^{*} & \\
& =\left[\max \left(x^{*}, y^{*}\right)\right]^{*} & \\
& =x^{* *} & \text { by Lemma 4.6 } \\
& =x &
\end{array}
$$

Thus, in all these cases, $x \sqsubseteq y$.

For the converse, suppose $x \sqsubseteq y$.

- Case 1: $x<0$. If $y \geq 0$ then $x<y$. So, we can assume $y<0$. Then

$$
\begin{array}{rlrl}
x & =x^{\prime} & & \text { since } x<0 \\
& =\left(x \Rightarrow y^{\prime}\right)^{\prime \prime} & & \text { by hypothesis } \\
& =x \Rightarrow y^{\prime} & & \text { by Lemma 4.5 } \\
& =x \Rightarrow y & & \\
& =\min (x, y) . &
\end{array}
$$

Hence $x \leq y$. 
- Case 2: $x \geq 0$. Suppose $y<0$. Then

$$
\begin{aligned}
x & =\left(x \Rightarrow y^{\prime}\right)^{\prime} \quad \text { by hypothesis } \\
& =(x \Rightarrow y)^{\prime} \\
& =\min (x, y)^{\prime} \\
& =y^{\prime} \\
& =y
\end{aligned}
$$

a contradiction. Hence $y \geq 0$. Consequently,

$$
\begin{array}{rlr}
x^{\prime} & =\left(x \Rightarrow y^{\prime}\right)^{\prime \prime} & \\
& =x \Rightarrow y^{\prime} \quad \text { by Lemma 4.5 } \\
& =\max \left(x^{\prime}, y^{\prime}\right),
\end{array}
$$

so, $x^{\prime} \geq y^{\prime}$. Then, by Lemma 4.5 and Lemma 4.6, $x=x^{\prime \prime} \leq y^{\prime \prime}=y$.

In view of Lemma 4.7 and Lemma 4.8, we have proved the following

Theorem $4.9[-\mathbf{n}, \mathbf{m}]$ is an $\mathbf{I}_{\mathbf{2}, \mathbf{0}}$-chain, where

$$
-n \sqsubset-n+1 \sqsubset \ldots \sqsubset-1 \sqsubset 0 \sqsubset 1 \sqsubset 2 \sqsubset \ldots \sqsubset m .
$$

\section{Characterization of finite $\mathbf{I}_{2,0}$-chains}

In this section we are going to prove our second main result. The following lemmas will be useful later in this section.

Lemma 5.1 Let $\mathbf{A} \in \mathbf{I}_{2,0}$. Then $0^{\prime}$ is the greatest element in $A$, relative to $\sqsubseteq$.

Proof Let $a \in A$. Since $\left(a \rightarrow(0 \rightarrow 0)^{\prime}\right)^{\prime}=\left(a \rightarrow 0^{\prime \prime}\right)^{\prime}=(a \rightarrow 0)^{\prime}=a^{\prime \prime}=a$, we have $a \sqsubseteq 0^{\prime}$.

Lemma 5.2 Let $\mathbf{A} \in \mathbf{I}_{2,0}$ and let $a, b \in A$ with $0 \sqsubseteq a \sqsubseteq b$. Then $b^{\prime} \sqsubseteq a^{\prime}$.

Proof

$$
\begin{array}{rlrl}
\left(b^{\prime} \rightarrow a^{\prime \prime}\right)^{\prime} & =\left(b^{\prime} \rightarrow a\right)^{\prime} & \\
& =\left(b^{\prime} \rightarrow\left(a \rightarrow b^{\prime}\right)^{\prime}\right)^{\prime} & & \text { by hypothesis } \\
& =\left(\left(a \rightarrow 0^{\prime}\right) \rightarrow b^{\prime \prime}\right)^{\prime} & & \text { by Lemma } 2.6 \text { (26) } \\
& =\left(\left(a \rightarrow 0^{\prime}\right) \rightarrow b\right)^{\prime} & \\
& =\left(\left(0 \rightarrow a^{\prime} \rightarrow b\right)^{\prime}\right. & & \text { by Lemma } 2.5 \text { (aa) } \\
& =\left(\left(0 \rightarrow a^{\prime}\right)^{\prime \prime} \rightarrow b\right)^{\prime} & \\
& =\left(0^{\prime} \rightarrow b\right)^{\prime} & & \text { by hypothesis } \\
& =b^{\prime} & & \text { by Lemma 2.4 (国). }
\end{array}
$$

Lemma 5.3 Let $\mathbf{A} \in \mathbf{I}_{2,0}$ and let $a \in A$. If $0 \sqsubseteq a$ then $0 \rightarrow a=0^{\prime}$. 
Proof First notice that, since $0 \sqsubseteq a, 0^{\prime}=\left(0 \rightarrow a^{\prime}\right)^{\prime \prime}=0 \rightarrow a^{\prime}$. Consequently,

(5.1) $0^{\prime}=0 \rightarrow a^{\prime}$.

Then

$$
\begin{aligned}
0^{\prime} & =0^{\prime} \rightarrow 0^{\prime} & & \text { by Lemma } 2.4 \text { (国) } \\
& =\left(0 \rightarrow a^{\prime} \rightarrow 0^{\prime}\right. & & \text { by (5.1) } \\
& =\left(0^{\prime} \rightarrow a^{\prime}\right) \rightarrow 0^{\prime} & & \text { by Lemma 2.6 (汭) } \\
& =a^{\prime} \rightarrow 0^{\prime} & & \text { by Lemma 2.4 (国) } \\
& =0 \rightarrow a . & & \text { by Lemma 2.5 (国) }
\end{aligned}
$$

Lemma 5.4 Let $\mathbf{A} \in \mathbf{I}_{2,0}$ and let $a, b \in A$. If $0 \sqsubseteq a$ and $0 \sqsubseteq b$ then $0 \sqsubseteq a \rightarrow b$.

Proof

$$
\begin{array}{rlrl}
{\left[0 \rightarrow(a \rightarrow b)^{\prime}\right]^{\prime}} & =\left[(a \rightarrow b) \rightarrow 0^{\prime}\right]^{\prime} & & \text { by Lemma } 2.5 \text { (国) } \\
& =(0 \rightarrow a) \rightarrow\left(b \rightarrow 0^{\prime}\right)^{\prime} & \text { by }(\mathrm{I}) \\
& =(0 \rightarrow a) \rightarrow\left(0 \rightarrow b^{\prime}\right)^{\prime} & & \text { by Lemma } 2.5 \text { (国) } \\
& =(0 \rightarrow a) \rightarrow 0 & & \text { since } 0 \sqsubseteq b \\
& =0^{\prime} \rightarrow 0 & & \text { by Lemma } 5.3 \text { since } 0 \sqsubseteq a \\
& =0 . & & \text { by Lemma } 2.4 \text { (国) }
\end{array}
$$

Corollary 5.5 Let $\mathbf{A} \in \mathbf{I}_{2,0}$ and $a \in A$. If $a \sqsupseteq 0$ then $a^{\prime} \sqsupseteq 0$.

Lemma 5.6 Let $\mathbf{A}$ be an $\mathbf{I}_{2,0}$-chain and let $a, b \in A$. Then $a^{\prime} \rightarrow b^{\prime}=b \rightarrow a$.

Proof Since $\mathbf{A}$ is a chain, we can assume that $b^{\prime} \sqsubseteq a$ or $a \sqsubseteq b^{\prime}$.

If $b^{\prime} \sqsubseteq a,\left(b^{\prime} \rightarrow a^{\prime}\right)^{\prime}=b^{\prime}$, then $b^{\prime} \rightarrow a^{\prime}=b$. Hence $b \rightarrow a=\left(b^{\prime} \rightarrow a^{\prime}\right) \rightarrow a=\left[\left(a^{\prime} \rightarrow b^{\prime}\right) \rightarrow\right.$ $\left.\left(a^{\prime} \rightarrow a\right)^{\prime}\right]^{\prime}$, using (I). By Lemma 2.4 (的), $\left[\left(a^{\prime} \rightarrow b^{\prime}\right) \rightarrow\left(a^{\prime} \rightarrow a\right)^{\prime}\right]^{\prime}=\left[\left(a^{\prime} \rightarrow b^{\prime}\right) \rightarrow a^{\prime}\right]^{\prime}=[[(a \rightarrow$ $\left.\left.\left.a^{\prime}\right) \rightarrow\left(b^{\prime} \rightarrow a^{\prime}\right)^{\prime}\right]^{\prime}\right]^{\prime}=\left(a \rightarrow a^{\prime}\right) \rightarrow\left(b^{\prime} \rightarrow a^{\prime}\right)^{\prime}=\left(a^{\prime \prime} \rightarrow a^{\prime}\right) \rightarrow\left(b^{\prime} \rightarrow a^{\prime}\right)^{\prime}=a^{\prime} \rightarrow b^{\prime}$.

If $a \sqsubseteq b^{\prime}$ then we have $a^{\prime}=\left(a \rightarrow b^{\prime \prime}\right)^{\prime \prime}=a \rightarrow b$, and the rest of the argument is similar to the previous case.

Lemma 5.7 Let $\mathbf{A}$ be $a \mathbf{I}_{2,0}$-chain with $|A| \geq 2$ and let $a \in A$ such that $a \sqsubset 0$. Then
(a) $0 \rightarrow a^{\prime}=a^{\prime}$
(b) $0 \rightarrow a=a$
(c) $(a \rightarrow a) \rightarrow a=a \rightarrow a$
(d) $a \rightarrow a=a^{\prime}$
(e) $a \rightarrow a=a$
(f) $a=a^{\prime}$.

\section{Proof}

(a) Since $a \sqsubseteq 0$, we have that $a=\left(a \rightarrow 0^{\prime}\right)^{\prime}$. Therefore, $a^{\prime}=\left(a \rightarrow 0^{\prime}\right)^{\prime \prime}=a \rightarrow 0^{\prime}=0 \rightarrow a^{\prime}$ by Lemma 2.5 (b). 
(b) Since $a \sqsubseteq 0$, we have

(5.2) $a=\left(a \rightarrow 0^{\prime}\right)^{\prime}$.

Then we get

$$
\begin{aligned}
(0 \rightarrow a) \rightarrow 0^{\prime} & =\left[(0 \rightarrow 0) \rightarrow\left(a \rightarrow 0^{\prime}\right)^{\prime}\right]^{\prime} & & \text { by }(\mathrm{I}) \\
& =[(0 \rightarrow 0) \rightarrow a]^{\prime} & & \text { by }(\underline{5.2}) \\
& =\left[0^{\prime} \rightarrow a\right]^{\prime} & & \\
& =a^{\prime} & & \text { by lemma } 2.4 \text { (国) }
\end{aligned}
$$

Using Lemma 2.5 (b), we obtain

$(5.3) a^{\prime}=0 \rightarrow(0 \rightarrow a)^{\prime}$.

Since $\mathbf{A}$ is a chain, $0 \sqsubseteq 0 \rightarrow a$ or $0 \rightarrow a \sqsubseteq 0$. Suppose that $0 \sqsubseteq 0 \rightarrow a$. Then $\left(0 \rightarrow(0 \rightarrow a)^{\prime}\right)^{\prime}=0$ Therefore, by (5.3),$a=a^{\prime \prime}=\left(0 \rightarrow(0 \rightarrow a)^{\prime}\right)^{\prime}=0$, a contradiction, since $a \neq 0$. Consequently, $0 \rightarrow a \sqsubseteq 0$. Hence, we have

$$
\begin{aligned}
0 \rightarrow a & =\left((0 \rightarrow a) \rightarrow 0^{\prime}\right)^{\prime} & & \text { since } 0 \rightarrow a \sqsubseteq 0 \\
& =\left(0 \rightarrow(0 \rightarrow a)^{\prime}\right)^{\prime} & & \text { by lemma 2.5 (b) } \\
& =a^{\prime \prime} & & \text { by (5.3) } \\
& =a . & &
\end{aligned}
$$

(IC)

$$
\begin{aligned}
& a \rightarrow a=(0 \rightarrow a) \rightarrow a \quad \text { by item (b) } \\
& =\left(a^{\prime} \rightarrow 0^{\prime}\right) \rightarrow a \quad \text { by lemma } 2.5 \text { (a) } \\
& =\left[\left(a^{\prime} \rightarrow a^{\prime}\right) \rightarrow\left(0^{\prime} \rightarrow a\right)^{\prime}\right]^{\prime} \quad \text { by }(\mathrm{I}) \\
& =\left[(a \rightarrow a) \rightarrow\left(0^{\prime} \rightarrow a\right)^{\prime}\right]^{\prime} \quad \text { by Lemma } 5.6 \\
& =\left[(a \rightarrow a) \rightarrow a^{\prime}\right]^{\prime} \quad \text { by lemma } 2.5 \text { (a) } \\
& =\left[\left[\left(a^{\prime \prime} \rightarrow a\right) \rightarrow\left(a \rightarrow a^{\prime}\right)^{\prime}\right]^{\prime}\right]^{\prime} \text { by (I) } \\
& =(a \rightarrow a) \rightarrow\left(a \rightarrow a^{\prime}\right)^{\prime} \\
& =(a \rightarrow a) \rightarrow\left(a^{\prime \prime} \rightarrow a^{\prime}\right)^{\prime} \\
& =(a \rightarrow a) \rightarrow a^{\prime \prime} \\
& =(a \rightarrow a) \rightarrow a \text {. }
\end{aligned}
$$

(dd) Since $\mathbf{A}$ is a chain, $0 \rightarrow a^{\prime} \sqsubseteq a$ or $a \sqsubseteq 0 \rightarrow a^{\prime}$.

First, we assume that $0 \rightarrow a^{\prime} \sqsubseteq a$. Then

$$
\begin{aligned}
a \rightarrow a & =(a \rightarrow a) \rightarrow a & & \text { by (I) } \\
& =\left(a^{\prime} \rightarrow a^{\prime}\right) \rightarrow a & & \text { by Lemma [5.6 } \\
& =a^{\prime} \rightarrow\left(a^{\prime} \rightarrow a^{\prime}\right)^{\prime} & & \text { by Lemma [5.6 } \\
& =(a \rightarrow 0) \rightarrow\left(a^{\prime} \rightarrow a^{\prime}\right)^{\prime} & & \\
& =\left[(a \rightarrow 0) \rightarrow\left(a^{\prime} \rightarrow a^{\prime}\right)^{\prime}\right]^{\prime \prime} & & \\
& =\left[\left(0 \rightarrow a^{\prime}\right) \rightarrow a^{\prime}\right]^{\prime} & & \text { using (I) } \\
& =0 \rightarrow a^{\prime} & & \text { since } 0 \rightarrow a^{\prime} \sqsubseteq a \\
& =a^{\prime} & & \text { using (国). }
\end{aligned}
$$

Next, we assume $a \sqsubseteq 0 \rightarrow a^{\prime}$, i.e., $\left(a \rightarrow\left(0 \rightarrow a^{\prime}\right)^{\prime}\right)^{\prime}=a$. Then, from (a) , we have $a \rightarrow a=\left(a \rightarrow a^{\prime \prime}\right)^{\prime \prime}=\left[\left(a \rightarrow\left(0 \rightarrow a^{\prime}\right)^{\prime}\right)^{\prime}\right]^{\prime}=a^{\prime}$. 
(还) Using the items (ㄷ), (dd) and Lemma 2.5 (d), we have $a \rightarrow a=(a \rightarrow a) \rightarrow a=a^{\prime} \rightarrow a=a$.

(If) This follows immediately from the two preceding items.

Lemma 5.8 Let $\mathbf{A}$ be an $\mathbf{I}_{2,0}$-chain with $|A| \geq 2$, and let $a, b \in A$. If $0 \sqsubseteq a$ and $b \sqsubset 0$ then $b \rightarrow a=b$ and $a \rightarrow b=b$.

Proof Since $0 \sqsubseteq a$ and $b \sqsubset 0$, we have that $\left(0 \rightarrow a^{\prime}\right)^{\prime}=0$ and $\left(b \rightarrow 0^{\prime}\right)^{\prime}=b$. Therefore, using Lemma 5.6, $b=b^{\prime \prime}=b^{\prime} \rightarrow 0=\left(b \rightarrow 0^{\prime}\right)^{\prime \prime} \rightarrow\left(0 \rightarrow a^{\prime}\right)^{\prime}=\left(b \rightarrow 0^{\prime}\right) \rightarrow\left(0 \rightarrow a^{\prime}\right)^{\prime}=\left(0 \rightarrow b^{\prime}\right) \rightarrow$ $\left(a \rightarrow 0^{\prime}\right)^{\prime}=\left[\left(b^{\prime} \rightarrow a\right) \rightarrow 0^{\prime}\right]^{\prime}$. Hence,

$(5.4) b=\left[\left(b^{\prime} \rightarrow a\right) \rightarrow 0^{\prime}\right]^{\prime}$.

From the hypothesis and Lemma $5.7(\mathbb{f})$, we have

(5.5) $b^{\prime}=b$.

Suppose that $0 \sqsubseteq b^{\prime} \rightarrow a$. Then $0=\left[0 \rightarrow\left(b^{\prime} \rightarrow a\right)^{\prime}\right]^{\prime}=\left[\left(b^{\prime} \rightarrow a\right) \rightarrow 0^{\prime}\right]^{\prime}$ by Lemma [5.6, implying $0=b$, which is a contradiction in view of (5.4). Consequently, $b^{\prime} \rightarrow a \sqsubseteq 0$, since $\mathbf{A}$ is a chain. Hence,

(5.6) $b^{\prime} \rightarrow a=\left[\left(b^{\prime} \rightarrow a\right) \rightarrow 0^{\prime}\right]^{\prime}$

From (5.4), (5.5) and (5.6) we conclude $b=b \rightarrow a$, proving the first half of the conclusion of the lemma. From

$$
\begin{aligned}
b & =\left(b \rightarrow a^{\prime}\right)^{\prime} \quad \text { since } b \sqsubseteq a, \text { as } 0 \sqsubseteq a \text { and } b \sqsubset 0 \\
& =\left(a^{\prime \prime} \rightarrow b^{\prime}\right)^{\prime} \quad \text { by Lemma } 5.6 \\
& =\left(a \rightarrow b^{\prime}\right)^{\prime} \\
& =(a \rightarrow b)^{\prime} \quad \text { by (5.5) }
\end{aligned}
$$

we conclude that $a \rightarrow b=b^{\prime}=b$ in view of (5.5), completing the second half.

Definition 5.9 Let $\mathbf{A}=\langle A ; \rightarrow, 0\rangle$ be a finite $\mathbf{I}_{2,0}$-chain. We let $A^{+}:=\{a \in A: a \sqsupset 0\}$ and $A^{-}:=\{a \in A: a \sqsubset 0\}$. Observe that $A=A^{+} \cup\{0\} \cup A^{-}$. Henceforth, without loss of generality, we will represent $A=[-n, m]$ with $0 \leq n, m \leq|A|-1$, such that

$$
-n \sqsubset-n+1 \sqsubset \ldots \sqsubset-1 \sqsubset 0 \sqsubset 1 \sqsubset 2 \sqsubset \ldots \sqsubset m \text {. }
$$

Remark 5.10 In view of the above definition, we can use the functions $*$ and $p$ of Definition 4.2 as functions on the domain $[-n, m]$ of $\mathbf{A}$ as well.

Now, we wish to prove that $\langle A ; \rightarrow, 0\rangle=\langle[-n ; m] ; \Rightarrow, 0\rangle$. To achieve this, we need the following lemmas.

Lemma 5.11 Let $\mathbf{A}=\langle A ; \rightarrow, 0\rangle$ be a finite $\mathbf{I}_{2,0}$-chain with $|A| \geq 2$. If $a \sqsupset 0$ then $a^{\prime}=p\left(p(a)^{\prime}\right)$.

Proof By hypothesis we have that $a \sqsupset 0$. Then $p(a) \sqsupseteq 0$. Hence $0 \sqsubseteq p(a) \sqsubset a$. Then, by Lemma 5.2,

(5.7) $a^{\prime} \sqsubseteq p(a)^{\prime}$. 
Since $a \sqsupset 0$, by Corollary [5.5, $a^{\prime} \sqsupseteq 0$. Therefore, by (5.7),

(5.8) $0 \sqsubseteq p(a)^{\prime}$.

If $a^{\prime}=p(a)^{\prime}$ then $a=p(a)$ and, consequently, $a=-n$, a contradiction, so $a^{\prime} \sqsubset p(a)^{\prime}$, and hence, $0 \sqsubseteq a^{\prime} \sqsubseteq p\left(p(a)^{\prime}\right) \sqsubset p(a)^{\prime}$. By lemma 5.2, $a \sqsupseteq\left[p\left(p(a)^{\prime}\right)\right]^{\prime} \sqsupseteq p(a)$. Thus

(5.9) $\left[p\left(p(a)^{\prime}\right)\right]^{\prime} \in\{a, p(a)\}$.

If $\left[p\left(p(a)^{\prime}\right)\right]^{\prime}=p(a)$, we have that $p\left(p(a)^{\prime}\right)=\left[p\left(p(a)^{\prime}\right)\right]^{\prime \prime}=p(a)^{\prime}$, a contradiction, since $p(a)^{\prime} \sqsupseteq 0$ by (5.8). Therefore $\left[p\left(p(a)^{\prime}\right)\right]^{\prime}=a$ and therefore, $p\left(p(a)^{\prime}\right)=a^{\prime}$.

Lemma 5.12 Let $\mathbf{A}=\langle A ; \rightarrow, 0\rangle$ be a finite $\mathbf{I}_{2,0}$-chain. If $a \in A$ then $a^{*}=a^{\prime}$.

Proof The statement $0^{\prime}=m=0^{*}$ follows from Lemma 5.1. If $a \sqsubset 0$ then $a^{\prime}=a$ by Lemma $5.7(\mathbb{f})$, and $a=a^{*}$ by definition, implying $a=a^{*}$.

Now assume that $a \sqsupset 0$. We will verify that $a^{\prime}=a^{*}$ by induction on $a$. If $a=1$, then, as $0^{\prime}=0^{*}$, we have, by Lemma 5.11, that $1^{\prime}=p\left(p(1)^{\prime}\right)=p\left(0^{\prime}\right)=p\left(0^{*}\right)=p\left(p(1)^{*}\right)=1^{*}$. The inductive hypothesis is that $p(a)^{\prime}=p(a)^{*}$. Hence, we have, by Lemma 5.11, $a^{\prime}=p\left(p(a)^{\prime}\right)=$ $p\left(p(a)^{*}\right)=a^{*}$.

The following theorem shows that the general method described in Definition 4.2 essentially gives all finite $\mathbf{I}_{2,0}$-chains.

Theorem 5.13 Let $\mathbf{A}$ be a finite $\mathbf{I}_{2,0}$-chain. Then $\mathbf{A} \cong\langle[-n, m] ; \Rightarrow, 0\rangle$ for some $0 \leq n, m \leq$ $|A|-1$.

Proof We will use the notation of Definition 5.9, Let $i, j \in A$. From Lemma 5.12, $i^{\prime}=i^{*}$ and $j^{\prime}=j^{*}$. It suffices to verify that

$$
i \rightarrow j= \begin{cases}\max \left(i^{\prime}, j\right) & \text { if } i, j \sqsupseteq 0 \\ \min (i, j) & \text { otherwise }\end{cases}
$$

with $0^{\prime}=m$. We consider the following cases:

- Case 1: $j>0$.

We need the following subcases:

- Case 1.1: $i>0$.

We make the following further subcases:

* Case 1.1.1: $i^{\prime} \geq j$.

Since $i^{\prime} \sqsupseteq j$, we observe that

(5.10) $\left(j \rightarrow i^{\prime \prime}\right)^{\prime}=j$.

Hence

$$
\begin{aligned}
i \rightarrow j & =i \rightarrow\left(j \rightarrow i^{\prime \prime}\right)^{\prime} & & \text { by (5.10) } \\
& =i \rightarrow(j \rightarrow i)^{\prime} & & \\
& =[(i \rightarrow j) \rightarrow i]^{\prime} & & \text { by Lemma 2.6 (4) } \\
& =[(0 \rightarrow j) \rightarrow i]^{\prime} & & \text { by Lemma 2.6 (515) } \\
& =\left[0^{\prime} \rightarrow i\right]^{\prime} & & \text { by Lemma 5.3 since } j \sqsupseteq 0 \\
& =i^{\prime} & & \text { by Lemma 2.4 (国) } \\
& =\max \left(i^{\prime}, j\right) & & \text { since } i^{\prime} \sqsupseteq j
\end{aligned}
$$


* Case 1.1.2: $i^{\prime}<j$.

Since $i^{\prime} \sqsubseteq j$, we have

(5.11) $\left(i^{\prime} \rightarrow j^{\prime}\right)^{\prime}=i^{\prime}$.

Therefore,

$$
\begin{aligned}
i \rightarrow j & =i^{\prime \prime} \rightarrow j & & \\
& =\left(i^{\prime} \rightarrow j^{\prime}\right)^{\prime \prime} \rightarrow j & & \text { by (15.11) } \\
& =\left(i^{\prime} \rightarrow j^{\prime}\right) \rightarrow j & & \\
& \left.=i^{\prime} \rightarrow 0^{\prime}\right) \rightarrow j & & \text { by Lemma 2.6 (11) } \\
& =(0 \rightarrow i) \rightarrow j & & \text { by Lemma 2.5 (回) } \\
& =0^{\prime} \rightarrow j & & \text { by Lemma [5.3 since } i \sqsupseteq 0 \\
& =j & & \text { by Lemma 2.4 (回) } \\
& =\max \left(i^{\prime}, j\right) & & \text { since } i^{\prime} \sqsubseteq j
\end{aligned}
$$

- Case 1.2: $i=0$.

Using Lemma 5.3 and Lemma 5.1, $0 \rightarrow j=0^{\prime}=\max \left(0^{\prime}, j\right)$.

- Case 1.3: $i<0$.

$$
\begin{aligned}
i \rightarrow j & =(0 \rightarrow i) \rightarrow j & & \text { by Lemma }[5.7(\mathbb{\mathrm { b }}) \\
& =\left(i^{\prime} \rightarrow 0^{\prime}\right) \rightarrow j & & \\
& =\left(i \rightarrow 0^{\prime}\right) \rightarrow j & & \text { by Lemma }[5.7(\mathbb{f}) \\
& =\left[\left(j^{\prime} \rightarrow i\right) \rightarrow\left(0^{\prime} \rightarrow j^{\prime}\right]^{\prime}\right. & & \text { by }(\mathrm{I}) \\
& =\left[\left(j^{\prime} \rightarrow i\right) \rightarrow j^{\prime}\right]^{\prime} & & \\
& =\left[(0 \rightarrow i) \rightarrow j^{\prime}\right]^{\prime} & & \text { by Lemma } 2.6(\mathbb{5}) \\
& =\left(i \rightarrow j^{\prime}\right)^{\prime} & & \text { by Lemma } 5.7(\underline{\mathrm{b}}) \\
& =i & & \text { since } i \sqsubset j \\
& =\min (i, j) & &
\end{aligned}
$$

- Case 2: $j<0$.

It is useful to consider the following subcases:

- Case 2.1: $i>0$

$$
\begin{aligned}
i \rightarrow j & =i \rightarrow j^{\prime} & & \text { by Lemma } 5.7(\mathbb{f}) \\
& =i \rightarrow\left(j \rightarrow i^{\prime}\right)^{\prime \prime} & & \text { since } j \sqsubset i \\
& =i \rightarrow\left(j \rightarrow i^{\prime}\right) & & \\
& =j \rightarrow i^{\prime} & & \text { by Lemma 2.6 (17) } \\
& =\left(j \rightarrow i^{\prime}\right)^{\prime \prime} & & \\
& =j^{\prime} & & \text { since } j \sqsubset i \\
& =j & & \text { by Lemma } 5.7(\mathbb{f}) \\
& =\min (i, j) & &
\end{aligned}
$$

- Case 2.2: $i=0$.

$$
\begin{aligned}
i \rightarrow j & =0 \rightarrow j \\
& =j \quad \text { by Lemma } 5.7 \text { (b) } \\
& =\min (i, j)
\end{aligned}
$$

- Case 2.3: $i<0$. 
* Case 2.3.1: $i \leq j$.

As $i \sqsubseteq j$, we have

(5.12) $\left(i \rightarrow j^{\prime}\right)^{\prime}=i$.

Observe

$$
\begin{aligned}
i \rightarrow j & =i \rightarrow j^{\prime} & & \text { by Lemma } 5.7(\mathbb{f}) \\
& =\left(i \rightarrow j^{\prime}\right)^{\prime \prime} & & \\
& =i^{\prime} & & \text { by }(15.12) \\
& =i & & \text { by Lemma } 5.7(\mathbb{f}) \\
& =\min (i, j) . & &
\end{aligned}
$$

* Case 2.3.2: $i>j$. We have

(5.13) $\left(j \rightarrow i^{\prime}\right)^{\prime}=j$.

as $j \sqsubseteq i$. Hence

$$
\begin{aligned}
i \rightarrow j & =j^{\prime} \rightarrow i^{\prime} & & \text { by Lemma } 5.6 \\
& =j \rightarrow i^{\prime} & & \text { by Lemma } 5.7(\mathbb{5}) \\
& =\left(j \rightarrow i^{\prime}\right)^{\prime \prime} & & \\
& =j^{\prime} & & \text { by }(\underline{5.13}) \\
& =j & & \text { by Lemma } 5.7 \text { (If) } \\
& =\min (j, i) & &
\end{aligned}
$$

- Case 3: $j=0$.

- Case 3.1: $i \geq 0$.

By Corollary [5.5, as $i \sqsupseteq 0$, we have that $i^{\prime}=i \rightarrow 0 \sqsupseteq 0$. Hence $i \rightarrow 0=i^{\prime}=$ $\max \left(i^{\prime}, 0\right)$.

- Case 3.2: $i<0$. We have that

$$
\begin{array}{rlr}
i \rightarrow j & =i \rightarrow 0 & \\
& =i^{\prime} & \\
& =i & \text { by Lemma } 5.7(\mathbb{I}) \\
& =\min (i, j) &
\end{array}
$$

Hence $\mathbf{A} \cong\langle[-n ; m] ; \Rightarrow, 0\rangle$.

The following theorem, our second main result, is now immediate from the preceding results.

Theorem 5.14 There are $n$ non-isomorphic $I_{2,0}$-chains of size $n$, for $n \in \mathbb{N}$.

\section{A Appendix: Proofs}

We would like to mention here that the identity: $x^{\prime \prime} \approx x$ is used in these proofs frequently without explicit mention.

Proof of Lemma 2.6. Items (11) to (17) are proved in [3]. The proofs of (18) to (26) are given in [5]. Let $a, b, c, d \in A$. 


$$
\begin{array}{rlrl}
(b \rightarrow c) \rightarrow a & =[(b \rightarrow c) \rightarrow a]^{\prime} \rightarrow[(b \rightarrow c) \rightarrow a] & & \text { by Lemma 2.4 (dd) } \\
& =\left[\left(a^{\prime} \rightarrow b\right) \rightarrow(c \rightarrow a)^{\prime}\right]^{\prime \prime} \rightarrow[(b \rightarrow c) \rightarrow a] & \text { from (I) } \\
& =\left[\left(a^{\prime} \rightarrow b\right) \rightarrow(c \rightarrow a)^{\prime}\right] \rightarrow[(b \rightarrow c) \rightarrow a] &
\end{array}
$$

(28)

$$
\begin{aligned}
{\left[\left[0 \rightarrow(a \rightarrow b)^{\prime}\right] \rightarrow\left(0 \rightarrow b^{\prime}\right)^{\prime}\right]^{\prime} } & =\left[\left\{0 \rightarrow(a \rightarrow b)^{\prime}\right\} \rightarrow\left(b \rightarrow 0^{\prime}\right)^{\prime}\right]^{\prime} & & \text { by Lemma 2.5 (国) } \\
& =\left[(a \rightarrow b)^{\prime} \rightarrow b\right] \rightarrow 0^{\prime} & & \text { by (I) } \\
& =0 \rightarrow\left[(a \rightarrow b)^{\prime} \rightarrow b\right]^{\prime} & & \text { by Lemma 2.5 (国) } \\
& =(a \rightarrow b) \rightarrow\left(0 \rightarrow b^{\prime}\right) & & \text { by (10) } \\
& =0 \rightarrow\left[(a \rightarrow b) \rightarrow b^{\prime}\right] & & \text { by (13) } \\
& =0 \rightarrow\left[\left(a \rightarrow 0^{\prime}\right) \rightarrow b^{\prime}\right] & & \text { by (1) } \\
& =\left(a \rightarrow 0^{\prime}\right) \rightarrow\left(0 \rightarrow b^{\prime}\right) & & \text { by (13) } \\
& =\left(0 \rightarrow a^{\prime}\right) \rightarrow\left(0 \rightarrow b^{\prime}\right) & & \text { by Lemma 2.5 (国) } \\
& =a^{\prime} \rightarrow\left(0 \rightarrow b^{\prime}\right) & & \text { by (20) } \\
& =\left[(0 \rightarrow a) \rightarrow\left(0 \rightarrow b^{\prime}\right)^{\prime}\right]^{\prime} & & \text { by (8) } \\
& =\left[(0 \rightarrow a) \rightarrow\left(b \rightarrow 0^{\prime}\right)^{\prime}\right]^{\prime} & & \text { by Lemma 2.5 (国) } \\
& =(a \rightarrow b) \rightarrow 0^{\prime} & & \text { by (I) } \\
& =0 \rightarrow(a \rightarrow b)^{\prime} & & \text { by Lemma 2.5 (国) }
\end{aligned}
$$

(29)

$$
\begin{array}{rlrl}
0 \rightarrow[(a \rightarrow b) \rightarrow c] & =0 \rightarrow\left[\left(c^{\prime} \rightarrow a\right) \rightarrow(b \rightarrow c)^{\prime}\right]^{\prime} & & \text { by }(\mathrm{I}) \\
& \sqsubseteq 0 \rightarrow(b \rightarrow c)^{\prime \prime} & & \text { by }(\underline{28}) \\
& =0 \rightarrow(b \rightarrow c) &
\end{array}
$$

(30)

$$
\begin{aligned}
& a^{\prime} \rightarrow\left(b \rightarrow 0^{\prime}\right)^{\prime}=(a \rightarrow 0) \rightarrow\left(b \rightarrow 0^{\prime}\right)^{\prime} \\
& =\left[\left\{\left(b \rightarrow 0^{\prime}\right) \rightarrow a\right\} \rightarrow\left\{0 \rightarrow\left(b \rightarrow 0^{\prime}\right)^{\prime}\right\}^{\prime}\right]^{\prime} \text { by (I) } \\
& =\left[\left\{\left(b \rightarrow 0^{\prime}\right) \rightarrow a\right\} \rightarrow\left\{0 \rightarrow\left(0 \rightarrow b^{\prime}\right)^{\prime}\right\}^{\prime}\right]^{\prime} \text { by Lemma 2.5 (a्a) } \\
& =\left[\left\{\left(b \rightarrow 0^{\prime}\right) \rightarrow a\right\} \rightarrow(0 \rightarrow b)^{\prime}\right]^{\prime} \quad \text { by (9) } \\
& =\left[\left\{\left(0 \rightarrow b^{\prime}\right) \rightarrow a\right\} \rightarrow(0 \rightarrow b)^{\prime}\right]^{\prime} \quad \text { by Lemma } 2.5 \text { (国) } \\
& =\left[\left[\left\{0 \rightarrow(0 \rightarrow b)^{\prime}\right\} \rightarrow a\right] \rightarrow(0 \rightarrow b)^{\prime}\right]^{\prime} \quad \text { by (9) } \\
& =\left[a \rightarrow(0 \rightarrow b)^{\prime}\right]^{\prime} \text { by (18) }
\end{aligned}
$$

(31)

$$
\begin{aligned}
{[(0 \rightarrow a) \rightarrow b]^{\prime} } & =[(b \rightarrow a) \rightarrow b]^{\prime} \quad \text { by (팜) } \\
& =\left[b \rightarrow(a \rightarrow b)^{\prime}\right]^{\prime \prime} \quad \text { by (14) } \\
& =b \rightarrow(a \rightarrow b)^{\prime}
\end{aligned}
$$

(32)

$$
\begin{array}{rlrl}
{\left[a \rightarrow\left(b \rightarrow 0^{\prime}\right)^{\prime}\right]^{\prime}} & =\left[a \rightarrow\left(0 \rightarrow b^{\prime}\right)^{\prime}\right]^{\prime} & \text { by Lemma } 2.5 \text { (国) } \\
& =a^{\prime} \rightarrow\left(b^{\prime} \rightarrow 0^{\prime}\right)^{\prime} & \text { by (30) } \\
& =a^{\prime} \rightarrow(0 \rightarrow b)^{\prime} & & \text { by Lemma } 2.5 \text { (国) }
\end{array}
$$

(33)

$$
\begin{aligned}
& b^{\prime} \rightarrow a^{\prime}=(b \rightarrow 0) \rightarrow a^{\prime} \\
& =\left[(a \rightarrow b) \rightarrow\left(0 \rightarrow a^{\prime}\right)^{\prime}\right]^{\prime} \quad \text { by }(\mathrm{I}) \\
& =\left[(a \rightarrow b) \rightarrow\left(a \rightarrow 0^{\prime}\right)^{\prime}\right]^{\prime} \text { by Lemma } 2.5 \text { (国) } \\
& =(a \rightarrow b)^{\prime} \rightarrow(0 \rightarrow a)^{\prime} \quad \text { by (32) with } x=a \rightarrow b, y=a
\end{aligned}
$$


(34)

$$
\begin{aligned}
& (0 \rightarrow a)^{\prime} \rightarrow(0 \rightarrow b)^{\prime}=[(0 \rightarrow a) \rightarrow 0] \rightarrow(0 \rightarrow b)^{\prime} \\
& =\left[\{(0 \rightarrow b) \rightarrow(0 \rightarrow a)\} \rightarrow\left\{0 \rightarrow(0 \rightarrow b)^{\prime}\right\}^{\prime}\right]^{\prime} \\
& \text { by }(\mathrm{I}) \\
& =\left[\left\{0 \rightarrow(0 \rightarrow b)^{\prime}\right\} \rightarrow(0 \rightarrow b)\right] \rightarrow\left[(0 \rightarrow a) \rightarrow\left\{0 \rightarrow(0 \rightarrow b)^{\prime}\right\}^{\prime}\right]^{\prime} \\
& \text { by }(\mathrm{I}) \\
& =\left[\left\{(0 \rightarrow b) \rightarrow(0 \rightarrow b)^{\prime}\right\} \rightarrow(0 \rightarrow b)\right] \rightarrow\left[(0 \rightarrow a) \rightarrow\left\{0 \rightarrow(0 \rightarrow b)^{\prime}\right\}^{\prime}\right]^{\prime} \\
& \text { by (5) } \\
& =\left[(0 \rightarrow b)^{\prime} \rightarrow(0 \rightarrow b)\right] \rightarrow\left[(0 \rightarrow a) \rightarrow\left\{0 \rightarrow(0 \rightarrow b)^{\prime}\right\}^{\prime}\right]^{\prime} \\
& \text { by Lemma } 2.4(\mathrm{~d}) \\
& =(0 \rightarrow b) \rightarrow\left[(0 \rightarrow a) \rightarrow\left\{0 \rightarrow(0 \rightarrow b)^{\prime}\right\}^{\prime}\right]^{\prime} \\
& \text { by Lemma } 2.4 \text { (d) } \\
& =(0 \rightarrow b) \rightarrow\left[(0 \rightarrow a) \rightarrow\left(0 \rightarrow b^{\prime}\right)^{\prime}\right]^{\prime} \\
& \text { by }(9) \\
& =(0 \rightarrow b) \rightarrow\left[(0 \rightarrow a) \rightarrow\left(b \rightarrow 0^{\prime}\right)^{\prime}\right]^{\prime} \\
& =(0 \rightarrow b) \rightarrow\left[(a \rightarrow b) \rightarrow 0^{\prime}\right] \\
& \text { by Lemma } 2.5 \text { (国) } \\
& \text { by (I) } \\
& =(0 \rightarrow b) \rightarrow\left[0 \rightarrow(a \rightarrow b)^{\prime}\right] \\
& =0 \rightarrow\left[(0 \rightarrow b) \rightarrow(a \rightarrow b)^{\prime}\right] \\
& \text { by Lemma } 2.5 \text { (国) } \\
& =0 \rightarrow(a \rightarrow b)^{\prime} \\
& =(a \rightarrow b) \rightarrow 0^{\prime} \\
& =\left[(0 \rightarrow a) \rightarrow\left(b \rightarrow 0^{\prime}\right)^{\prime}\right]^{\prime} \\
& \text { by Lemma 2.5 (国) } \\
& \text { by (13) } \\
& \text { by (3) } \\
& =\left[(0 \rightarrow a) \rightarrow\left(0 \rightarrow b^{\prime}\right)^{\prime} \quad \text { by Lemma } 2.5\right. \text { (国) } \\
& =\left[a^{\prime} \rightarrow\left(0 \rightarrow b^{\prime}\right)\right]^{\prime \prime} \\
& =a^{\prime} \rightarrow\left(0 \rightarrow b^{\prime}\right) \\
& =0 \rightarrow\left(a^{\prime} \rightarrow b^{\prime}\right) \quad \text { by (13) } \text {. }
\end{aligned}
$$

(35)

$$
\begin{aligned}
{\left[(a \rightarrow b)^{\prime} \rightarrow\left\{b \rightarrow(a \rightarrow b)^{\prime}\right\}^{\prime}\right]^{\prime} } & =\left[(a \rightarrow b)^{\prime} \rightarrow b\right] \rightarrow(a \rightarrow b)^{\prime} & & \text { by (41) } \\
& =(0 \rightarrow b) \rightarrow(a \rightarrow b)^{\prime} & & \text { by (15) } \\
& =(a \rightarrow b)^{\prime} & & \text { by (3) }
\end{aligned}
$$

(36)

$$
\begin{aligned}
& (0 \rightarrow a) \rightarrow b=(b \rightarrow a) \rightarrow b \\
& \text { by (5) } \\
& =\left[b \rightarrow(a \rightarrow b)^{\prime}\right]^{\prime} \\
& \sqsubseteq(a \rightarrow b)^{\prime} \rightarrow\left[b \rightarrow(a \rightarrow b)^{\prime}\right]^{\prime} \\
& =\left[\left\{(a \rightarrow b)^{\prime} \rightarrow b\right\} \rightarrow(a \rightarrow b)^{\prime}\right]^{\prime} \\
& =\left[(0 \rightarrow b) \rightarrow(a \rightarrow b)^{\prime}\right]^{\prime} \\
& =(a \rightarrow b)^{\prime \prime} \\
& =a \rightarrow b \\
& \text { by (44) } \\
& \text { by (35) with } x=b, y=(a \rightarrow b)^{\prime} \\
& \text { by (41) } \\
& \text { by (15) } \\
& \text { by (3) } \\
& \text { since } x^{\prime \prime} \approx x
\end{aligned}
$$


(37)

$$
\begin{array}{rll}
{\left[\left\{a \rightarrow(b \rightarrow a)^{\prime}\right\} \rightarrow a^{\prime \prime}\right]^{\prime}} & =\left[\left\{a \rightarrow(b \rightarrow a)^{\prime}\right\} \rightarrow a\right]^{\prime} & \\
& =\left[\left\{0 \rightarrow(b \rightarrow a)^{\prime}\right\} \rightarrow a\right]^{\prime} & \text { by (15) } \\
& =\left[\left\{(b \rightarrow a) \rightarrow 0^{\prime}\right\} \rightarrow a\right]^{\prime} & \text { by Lemma 2.5 (国) } \\
& =\left[\left\{(b \rightarrow a) \rightarrow a^{\prime}\right\} \rightarrow a\right]^{\prime} & \text { by (1) } \\
& =\left[\left\{\left(b \rightarrow 0^{\prime}\right) \rightarrow a^{\prime}\right\} \rightarrow a\right]^{\prime} & \text { by (1) } \\
& =\left[\left\{\left(b \rightarrow 0^{\prime}\right) \rightarrow 0^{\prime}\right\} \rightarrow a\right]^{\prime} & \text { by (1) } \\
& =\left[\left\{\left(b \rightarrow 0^{\prime \prime} \rightarrow 0^{\prime}\right\} \rightarrow a\right]^{\prime}\right. & \text { by (1) } \\
& =\left[\left\{(b \rightarrow 0) \rightarrow 0^{\prime}\right\} \rightarrow a\right]^{\prime} & \\
& =\left[\left(b^{\prime} \rightarrow 0^{\prime} \rightarrow a\right]^{\prime}\right. & \\
& =[(0 \rightarrow b) \rightarrow a]^{\prime} & \\
& =[(a \rightarrow b) \rightarrow a]^{\prime} & \text { by Lemma } 2.5 \text { (1) } \\
& =a \rightarrow(b \rightarrow a)^{\prime} & \text { by (4) }
\end{array}
$$

\section{Proof of Lemma 3.3}

(11) Observe that by Lemma 2.5 (国), Lemma 2.6 (11) and the hypothesis we have that $(0 \rightarrow$ $\left.a^{\prime}\right) \rightarrow b=\left(a \rightarrow 0^{\prime}\right) \rightarrow b=\left(a \rightarrow b^{\prime}\right) \rightarrow b=\left(a \rightarrow b^{\prime}\right)^{\prime \prime} \rightarrow b=a^{\prime} \rightarrow b$.

(21)

$$
\begin{aligned}
b \rightarrow a^{\prime} & =\left[\left(0 \rightarrow a^{\prime}\right) \rightarrow b\right] \rightarrow a^{\prime} & & \text { by Lemma 2.6 (18) } \\
& =\left(a^{\prime} \rightarrow b\right) \rightarrow a^{\prime} & & \text { from (1) } \\
& =(0 \rightarrow b) \rightarrow a^{\prime} & & \text { by Lemma 2.6 (15) }
\end{aligned}
$$

(3)

$$
\begin{array}{rlrl}
b \rightarrow a^{\prime} & =(0 \rightarrow b) \rightarrow a^{\prime} & & \text { from (2) } \\
& =(0 \rightarrow b) \rightarrow\left(a \rightarrow b^{\prime}\right)^{\prime \prime} & & \text { by hypothesis } \\
& =(0 \rightarrow b) \rightarrow\left(a \rightarrow b^{\prime}\right) & \\
& =\left(0 \rightarrow b^{\prime \prime}\right) \rightarrow\left(a \rightarrow b^{\prime}\right) & \\
& =a \rightarrow b^{\prime} & & \text { by Lemma } 2.6 \\
& =\left(a \rightarrow b^{\prime}\right)^{\prime \prime} & & \\
& =a^{\prime} & & \text { by hypothesis }
\end{array}
$$

(4)

$$
\begin{aligned}
0 \rightarrow\left(a^{\prime} \rightarrow b\right) & =a^{\prime} \rightarrow(0 \rightarrow b) & & \text { by Lemma 2.6 (13) } \\
& =0 \rightarrow\left(a \rightarrow b^{\prime}\right)^{\prime} & & \text { by Lemma 2.6 (10) } \\
& =0 \rightarrow a & & \text { by hypothesis }
\end{aligned}
$$

(5) By hypothesis and (I) we have that $(d \rightarrow a) \rightarrow b^{\prime}=\left[(b \rightarrow d) \rightarrow\left(a \rightarrow b^{\prime}\right)^{\prime}\right]^{\prime}=[(b \rightarrow d) \rightarrow$ $a]^{\prime}$.

(6)

$$
\begin{aligned}
{\left[\left\{d \rightarrow\left(0 \rightarrow b^{\prime}\right)\right\} \rightarrow a\right]^{\prime} } & =\left(a^{\prime} \rightarrow d\right) \rightarrow\left[\left(0 \rightarrow b^{\prime}\right) \rightarrow a\right]^{\prime} & & \text { by (I) } \\
& =\left(a^{\prime} \rightarrow d\right) \rightarrow\left[\left(a \rightarrow b^{\prime}\right) \rightarrow a\right]^{\prime} & & \text { by Lemma 2.6 (5) } \\
& =\left(a^{\prime} \rightarrow d\right) \rightarrow\left[\left(a \rightarrow b^{\prime}\right)^{\prime \prime} \rightarrow a\right]^{\prime} & & \\
& =\left(a^{\prime} \rightarrow d\right) \rightarrow\left(a^{\prime} \rightarrow a\right)^{\prime} & & \text { by hypothesis } \\
& =\left(a^{\prime} \rightarrow d\right) \rightarrow a^{\prime} & & \text { by Lemma 2.4 (d) } \\
& =\left(a^{\prime} \rightarrow d\right) \rightarrow\left(0^{\prime} \rightarrow a\right)^{\prime} & & \text { by Lemma 2.4 (国) } \\
& =\left[\left(d \rightarrow 0^{\prime}\right) \rightarrow a\right]^{\prime} & & \text { by (I) } \\
& =(0 \rightarrow d) \rightarrow a^{\prime} & & \text { by Lemma 2.6 (11) }
\end{aligned}
$$


(7)

$$
\begin{aligned}
a \rightarrow\left[\left(a^{\prime} \rightarrow d\right) \rightarrow\left\{(0 \rightarrow a) \rightarrow b^{\prime}\right\}\right]= & a \rightarrow\left[\left(a^{\prime} \rightarrow d\right) \rightarrow\left\{(b \rightarrow 0) \rightarrow\left(a \rightarrow b^{\prime}\right)^{\prime}\right\}^{\prime}\right] \\
& \text { by }(\mathrm{I}) \\
= & a \rightarrow\left[\left(a^{\prime} \rightarrow d\right) \rightarrow\{(b \rightarrow 0) \rightarrow a\}^{\prime}\right] \\
& \text { by hypothesis } \\
= & a \rightarrow[\{d \rightarrow(b \rightarrow 0)\} \rightarrow a]^{\prime} \\
& \text { by }(\mathrm{I}) \\
= & {[[d \rightarrow(0 \rightarrow(b \rightarrow 0))] \rightarrow a]^{\prime} } \\
& \text { by Lemma } 2.6(\underline{22}) \text { with } x=d, y=b \rightarrow 0, z=a \\
= & {\left[\left[d \rightarrow\left(0 \rightarrow b^{\prime}\right)\right] \rightarrow a\right]^{\prime} } \\
= & (0 \rightarrow d) \rightarrow a^{\prime} \\
& \text { by (6) }
\end{aligned}
$$

(8)

$$
\begin{aligned}
a \rightarrow\left((d \rightarrow a) \rightarrow b^{\prime}\right) & =a \rightarrow[(b \rightarrow d) \rightarrow a]^{\prime} & & \\
& =a^{\prime \prime} \rightarrow[(b \rightarrow d) \rightarrow a]^{\prime} & & \\
& =\left(a^{\prime} \rightarrow 0\right) \rightarrow[(b \rightarrow d) \rightarrow a]^{\prime} & & \\
& =[\{0 \rightarrow(b \rightarrow d)\} \rightarrow a]^{\prime} & & \\
& =\left[\left\{(b \rightarrow d)^{\prime} \rightarrow 0^{\prime}\right\} \rightarrow a\right]^{\prime} & & \\
& =\left[\left\{((b \rightarrow d) \rightarrow 0) \rightarrow 0^{\prime}\right\} \rightarrow a\right]^{\prime} & & \\
& \left.=\left[\left\{(b \rightarrow d) \rightarrow 0^{\prime}\right) \rightarrow 0^{\prime}\right\} \rightarrow a\right]^{\prime} & & \text { by Lemma 2.6 (1) } \\
& =\left[\left\{\left((b \rightarrow d) \rightarrow 0^{\prime}\right) \rightarrow a^{\prime}\right\} \rightarrow a\right]^{\prime} & & \text { by Lemma 2.6 (1) } \\
& =\left[\left\{((b \rightarrow d) \rightarrow a) \rightarrow a^{\prime}\right\} \rightarrow a\right]^{\prime} & & \text { by Lemma 2.6 (1) } \\
& =\left[\left\{((b \rightarrow d) \rightarrow a) \rightarrow 0^{\prime}\right\} \rightarrow a\right]^{\prime} & & \\
& =\left[\left\{0 \rightarrow((b \rightarrow d) \rightarrow a)^{\prime}\right\} \rightarrow a\right]^{\prime} & & \\
& =\left[\left\{0 \rightarrow\left((d \rightarrow a) \rightarrow b^{\prime}\right)\right\} \rightarrow a\right]^{\prime} & & \text { by Lemma (15) (1) } \\
& =\left[\left\{a \rightarrow\left((d \rightarrow a) \rightarrow b^{\prime}\right)\right\} \rightarrow a\right]^{\prime} & & \\
& =\left(a^{\prime} \rightarrow a\right) \rightarrow\left[\left\{(d \rightarrow a) \rightarrow b^{\prime}\right\} \rightarrow a\right]^{\prime} & & \text { by Lemma 2.6 (15) } \\
& =a \rightarrow\left[\left\{(d \rightarrow a) \rightarrow b^{\prime}\right\} \rightarrow a\right]^{\prime} & & \text { by (I) } \\
& =a \rightarrow\left[\left\{a^{\prime} \rightarrow(d \rightarrow a)\right\} \rightarrow\left(b^{\prime} \rightarrow a\right)^{\prime}\right] & & \\
& =a \rightarrow\left[\left\{a^{\prime} \rightarrow(d \rightarrow a)\right\} \rightarrow\{(b \rightarrow 0) \rightarrow a\}^{\prime}\right] & & \\
& =a \rightarrow\left[\left\{a^{\prime} \rightarrow(d \rightarrow a)\right\} \rightarrow\left\{(b \rightarrow 0) \rightarrow\left(a \rightarrow b^{\prime}\right)^{\prime}\right\}^{\prime}\right] & & \text { by hypothesis } \\
& =a \rightarrow\left[\left\{a^{\prime} \rightarrow(d \rightarrow a)\right\} \rightarrow\left\{(0 \rightarrow a) \rightarrow b^{\prime}\right\}\right] & & \text { by (I) } \\
& =[0 \rightarrow(d \rightarrow a)] \rightarrow a^{\prime} & & \text { by (7) with } d:=d \rightarrow a \\
& =a \rightarrow(d \rightarrow a)^{\prime} & & \text { by Lemma 2.6 (23) }
\end{aligned}
$$

(9)

$$
\begin{aligned}
{[0 \rightarrow(b \rightarrow d)] \rightarrow a } & =\left[\left(a^{\prime} \rightarrow 0\right) \rightarrow((b \rightarrow d) \rightarrow a)^{\prime}\right]^{\prime} & & \text { by (I) } \\
& =\left[a \rightarrow((b \rightarrow d) \rightarrow a)^{\prime}\right]^{\prime} & & \\
& =\left[a \rightarrow\left((d \rightarrow a) \rightarrow b^{\prime}\right)\right]^{\prime} & & \text { by (5) } \\
& =\left[a \rightarrow(d \rightarrow a)^{\prime}\right]^{\prime} & & \text { by (8) } \\
& =(a \rightarrow d) \rightarrow a & & \text { by Lemma 2.6 (4) } \\
& =(0 \rightarrow d) \rightarrow a & & \text { by Lemma 2.6 (15) }
\end{aligned}
$$


(10)

$$
\begin{aligned}
(b \rightarrow(a \rightarrow d)) \rightarrow a & =\left[\left(a^{\prime} \rightarrow b\right) \rightarrow\{(a \rightarrow d) \rightarrow a\}^{\prime}\right]^{\prime} & & \text { by (I) } \\
& =\left[\left(a^{\prime} \rightarrow b\right) \rightarrow\{(0 \rightarrow d) \rightarrow a\}^{\prime}\right]^{\prime} & & \text { by Lemma 2.6 (15) } \\
& =[b \rightarrow(0 \rightarrow d)] \rightarrow a & & \text { by (I) } \\
& =[0 \rightarrow(b \rightarrow d)] \rightarrow a & & \text { by Lemma 2.6 (13) } \\
& =(0 \rightarrow d) \rightarrow a & & \text { by (9) }
\end{aligned}
$$

(11)

$$
\begin{aligned}
b \rightarrow\left(0 \rightarrow a^{\prime}\right) & =(0 \rightarrow b) \rightarrow\left(0 \rightarrow a^{\prime}\right) & & \text { by Lemma 2.6 (20) } \\
& =0 \rightarrow\left[(0 \rightarrow b) \rightarrow a^{\prime}\right] & & \text { by Lemma 2.6 (13) } \\
& =0 \rightarrow\left[(a \rightarrow 0) \rightarrow\left(b \rightarrow a^{\prime}\right)^{\prime}\right]^{\prime} & & \text { by }(\mathrm{I}) \\
& =0 \rightarrow\left[a^{\prime} \rightarrow\left(b \rightarrow a^{\prime}\right)^{\prime}\right]^{\prime} & & \\
& =0 \rightarrow\left(a^{\prime} \rightarrow a^{\prime \prime}\right)^{\prime} & & \text { by (3) } \\
& =0 \rightarrow\left(a^{\prime} \rightarrow a\right)^{\prime} & & \\
& =0 \rightarrow a^{\prime} & & \text { by Lemma 2.4 (d) }
\end{aligned}
$$

(12) From (I) and by hypothesis we have that $\left[(d \rightarrow a) \rightarrow b^{\prime}\right]^{\prime}=(b \rightarrow d) \rightarrow\left(a \rightarrow b^{\prime}\right)^{\prime}=(b \rightarrow$ d) $\rightarrow a$.

(13)

$$
\begin{aligned}
a^{\prime} \rightarrow b & =\left(a \rightarrow b^{\prime}\right) \rightarrow b & & \text { by hypothesis } \\
& =\left[\left(b^{\prime} \rightarrow a\right) \rightarrow\left(b^{\prime} \rightarrow b\right)^{\prime}\right]^{\prime} & & \text { using (I) } \\
& =\left[\left(b^{\prime} \rightarrow a\right) \rightarrow b^{\prime}\right]^{\prime} & & \text { by Lemma 2.4 (dd) } \\
& =b^{\prime} \rightarrow\left(a \rightarrow b^{\prime}\right)^{\prime} & & \text { by Lemma 2.6 (44) } \\
& =b^{\prime} \rightarrow a & & \text { by hypothesis }
\end{aligned}
$$


(14)

$$
\begin{aligned}
& \left(d \rightarrow 0^{\prime}\right) \rightarrow\left(a^{\prime} \rightarrow b\right)=\left(a^{\prime} \rightarrow b\right)^{\prime} \rightarrow\left[\left(d \rightarrow 0^{\prime}\right) \rightarrow\left(a^{\prime} \rightarrow b\right)\right] \\
& \text { by Lemma 2.6 (17) } \\
& =\left(a^{\prime} \rightarrow b\right)^{\prime} \rightarrow\left[\left(d \rightarrow 0^{\prime}\right) \rightarrow\left(b^{\prime} \rightarrow a\right)\right] \\
& \text { by (13) } \\
& =\left(a^{\prime} \rightarrow b\right)^{\prime} \rightarrow\left[\left(d \rightarrow 0^{\prime}\right) \rightarrow\left\{(0 \rightarrow a) \rightarrow b^{\prime}\right\}^{\prime}\right] \\
& \text { by (12) with } d=0 \\
& =\left(a^{\prime} \rightarrow b\right)^{\prime} \rightarrow\left[\left\{(0 \rightarrow a) \rightarrow b^{\prime}\right\} \rightarrow\left\{d \rightarrow\left((0 \rightarrow a) \rightarrow b^{\prime}\right)\right\}^{\prime}\right] \\
& \text { by Lemma } 2.6 \text { (26) with } \\
& x=(0 \rightarrow a) \rightarrow b^{\prime}, y=d \\
& =\left(a^{\prime} \rightarrow b\right)^{\prime} \rightarrow\left[\left(b^{\prime} \rightarrow a\right)^{\prime} \rightarrow\left\{d \rightarrow\left((0 \rightarrow a) \rightarrow b^{\prime}\right)\right\}^{\prime}\right] \\
& \text { by (12) with } d=0 \\
& =\left(a^{\prime} \rightarrow b\right)^{\prime} \rightarrow\left[\left(a^{\prime} \rightarrow b\right)^{\prime} \rightarrow\left\{d \rightarrow\left((0 \rightarrow a) \rightarrow b^{\prime}\right)\right\}^{\prime}\right] \\
& \text { by }(13) \\
& =\left(a^{\prime} \rightarrow b\right)^{\prime} \rightarrow\left[d \rightarrow\left\{(0 \rightarrow a) \rightarrow b^{\prime}\right\}\right]^{\prime} \\
& \text { by Lemma } 2.6 \text { (21) } \\
& =\left(b^{\prime} \rightarrow a\right)^{\prime} \rightarrow\left[d \rightarrow\left\{(0 \rightarrow a) \rightarrow b^{\prime}\right\}\right]^{\prime} \\
& \text { by (13) } \\
& =\left[(0 \rightarrow a) \rightarrow b^{\prime}\right] \rightarrow\left[d \rightarrow\left\{(0 \rightarrow a) \rightarrow b^{\prime}\right\}\right]^{\prime} \\
& =\left[(0 \rightarrow d) \rightarrow\left\{(0 \rightarrow a) \rightarrow b^{\prime}\right\}\right]^{\prime} \\
& \text { by Lemma 2.6 (31) with } \\
& =\left[\left(b^{\prime} \rightarrow d\right) \rightarrow\left\{(0 \rightarrow a) \rightarrow b^{\prime}\right\}\right]^{\prime} \\
& x=d, y=(0 \rightarrow a) \rightarrow b^{\prime} \\
& \text { by Lemma } 2.6 \text { (15) with } \\
& =\left[\left(b^{\prime} \rightarrow d\right) \rightarrow\left(b^{\prime} \rightarrow a\right)^{\prime}\right]^{\prime} \\
& x=b, y=d, z=a \\
& =\left[\left(b^{\prime} \rightarrow d\right) \rightarrow\left(a^{\prime} \rightarrow b\right)^{\prime}\right]^{\prime} \\
& \text { by (12) with } d=0 \\
& =\left(d \rightarrow a^{\prime}\right) \rightarrow b \quad \text { by (I). }
\end{aligned}
$$

(15)

$$
\begin{aligned}
{\left[\left(0 \rightarrow a^{\prime}\right) \rightarrow b\right]^{\prime} } & =\left[\left(a \rightarrow 0^{\prime}\right) \rightarrow b\right]^{\prime} \quad \text { by Lemma } 2.5 \text { (国) } \\
& =(0 \rightarrow a) \rightarrow b^{\prime} \quad \text { by Lemma 2.6 (11) }
\end{aligned}
$$

(16)

$$
\begin{array}{rll}
\left(a^{\prime} \rightarrow b\right)^{\prime} & =\left[\left(0 \rightarrow a^{\prime}\right) \rightarrow b\right]^{\prime} & \text { by }(11) \\
& =(0 \rightarrow a) \rightarrow b^{\prime} & \text { by }(15)
\end{array}
$$


(17)

$$
\begin{aligned}
& {\left[\left\{b^{\prime} \rightarrow((b \rightarrow d) \rightarrow a)\right\} \rightarrow(0 \rightarrow b)^{\prime}\right]^{\prime}=\left[\left\{b^{\prime} \rightarrow\left((d \rightarrow a) \rightarrow b^{\prime}\right)^{\prime}\right\} \rightarrow(0 \rightarrow b)^{\prime}\right]^{\prime}} \\
& \text { by (12) } \\
& =\left[\left\{(d \rightarrow a) \rightarrow b^{\prime}\right\}^{\prime} \rightarrow 0\right] \rightarrow b \\
& =\left[(d \rightarrow a) \rightarrow b^{\prime}\right] \rightarrow b \\
& =b^{\prime} \rightarrow\left[(d \rightarrow a) \rightarrow b^{\prime}\right]^{\prime} \\
& \text { by (I) } \\
& \text { by Lemma } 2.6 \text { (14) with } \\
& =b^{\prime} \rightarrow[(b \rightarrow d) \rightarrow a] \quad \text { by (12). }
\end{aligned}
$$

\section{References}

[1] R. Balbes and PH. Dwinger, Distributive lattices, Univ. of Missouri Press, Columbia, 1974.

[2] S. Burris and H. P. Sankappanavar, A course in universal algebra, Springer-Verlag, New York, 1981. The free, corrected version (2012) is available online as a PDF file at math.uwaterloo.ca/ snburris. It is also available for a free download at Sankappanavar's profile page at www.researchgate.net.

[3] J. M. Cornejo and H. P. Sankappanavar, Implication Zroupoids I. Submitted for publication (2015).

[4] J. M. Cornejo and H. P. Sankappanavar, Implication Zroupoids II. In Preparation.

[5] J. M. Cornejo and H. P. Sankappanavar, Semisimple Varieties of Implication Zroupoids. Submitted for publication (2015).

[6] W. McCune, Prover9 and Mace 4, http://www.cs.unm.edu/mccune/prover9/

[7] H. P. Sankappanavar, De Morgan algebras: New perspectives and applications, Scientia Mathematica Japonica 75(1): 21-50, 2012. 